\title{
Comment évaluer la productivité et l'efficacité des hôpitaux publics et privés ? Les enjeux de la convergence tarifaire
}

\author{
Brigitte Dormont* et Carine Milcent**1
}

\begin{abstract}
Résumé
En France les cliniques privées jouent un rôle important dans l'offre de soins hospitaliers. En 2007, $56 \%$ des séjours ont eu lieu dans des hôpitaux publics, 8 \% dans des hôpitaux privés à but non lucratifs, qui participent au service public hospitalier (PSPH) et $36 \%$ dans des hôpitaux privés à but lucratif (cliniques). Plusieurs rapports administratifs ont récemment montré qu'un séjour dans un hôpital public ou PSPH était plus coûteux que dans une clinique privée, suggérant que la productivité du secteur public était relativement faible. Cet article a pour but de comprendre les différences de productivité observées en France entre les hôpitaux publics, les hôpitaux PSPH et les cliniques privées.
\end{abstract}

L'introduction de la Tarification à l'Activité (T2A) en 2004 visait à améliorer l'efficacité de la dépense pour les soins hospitaliers. La mise en œuvre du nouveau paiement est progressive, avec une application intégrale à partir de 2008. Dès le départ, les tarifs différaient selon que le séjour avait lieu dans un hôpital public ou un hôpital privé à but lucratif. Actuellement, les paiements par séjour dans une pathologie donnée sont en moyenne $27 \%$ plus élevés dans le secteur public que dans le secteur privé. Une convergence des grilles tarifaires des secteurs public (et PSPH) et privé est prévue à I'horizon 2018. Mettre en place cette convergence revient à supposer que les différences de coûts sont exclusivement dues à des différences d'efficacité, qui seraient éliminées par l'introduction d'une concurrence entre les deux secteurs.

Notre objectif est d'examiner s'il existe une influence de la composition des séjours sur la productivité des hôpitaux en matière de soins aigus. Si tel est le cas, introduire de la concurrence entre les établissements sur la base de la T2A crée de fortes pressions en faveur d'une réorganisation de l'offre de soins. Ces changements sont souhaitables s'ils permettent d'améliorer l'efficacité dans la délivrance des soins hospitaliers. En revanche, il n'est pas souhaitable que le système de tarification crée des incitations à la sélection de patients ou à l'arrêt de la production de soins qui seraient importants du point de vue du bien-être collectif.

Les données utilisées proviennent de deux bases administratives: les données du PMSI et celles de la SAE. La base finale contient 1604 hôpitaux sur la période 1998-2003, dont 642 sont publics, 126 sont PSPH et 836 sont privés. Pour les soins aigus cette base est proche de l'exhaustivité: en 2003, elle représente $90 \%$ de l'ensemble des séjours de soins aigus en France métropolitaine. L'analyse couvre

\footnotetext{
* Université Paris Dauphine (Leda-Legos) et Cepremap.

** PSE et Cepremap.

${ }^{1}$ Les auteures remercient pour leurs commentaires les participants du Workshop on Health, inequalities, risk and public policy, Paris Descartes, Septembre 2009, du séminaire " Economie de la santé » de PSE, février 2010 et du séminaire 3S, DREES, mai 2010. Elles expriment leur reconnaissance à Victoria Verdy pour son travail d'assistant de recherche et à la Drees pour son aide dans l'accès aux données. Ce travail a bénéficié d'un soutien financier du Cepremap et de la Fondation du Risque (Chaire Santé, risque, assurance, Allianz).
} 
les six années précédant l'introduction de la T2A en France afin d'observer précisément la situation qui préexistait avant la mise en place de nouvelles incitations. Ce travail permet d'avoir un référentiel sur la situation du tissu hospitalier français et les performances comparées des établissements publics, PSPH et privés, avant la mise en place de la réforme.

En synthétisant l'activité "multiproduit" de l'hôpital par un produit homogène défini selon des critères identiques, quel que soit le statut de l'hôpital, nous montrons que le diagnostic sur l'efficacité productive des hôpitaux publics dépend de la définition de la frontière de production: lorsque l'on utilise une fonction de production classique mettant en relation les inputs et l'output, les scores d'efficacité des hôpitaux publics sont inférieurs à ceux des hôpitaux PSPH, eux-mêmes inférieurs à ceux des cliniques privées. Mais l'ordre des performances relatives s'inverse lorsque l'on tient compte des caractéristiques de la patientèle et la composition des séjours des hôpitaux : à l'exception des petits établissements, les hôpitaux publics et PSPH apparaissent alors plus efficaces que les cliniques privées.

Ces résultats doivent être interprétés à la lumière des différences de cahiers des charges encadrant les activités des hôpitaux publics et privés. Il est frappant de constater que la prise en compte d'indicateurs concernant la structure de l'activité et la composition de la patientèle modifie radicalement le diagnostic sur l'efficacité productive des hôpitaux publics.

La plus faible productivité des hôpitaux publics s'explique principalement par le nombre de lits, des personnels médico-techniques en sureffectifs, la composition de leur patientèle et celle de leurs séjours (caractérisée entre autres par une faible proportion de séjours chirurgicaux). Elle ne s'explique pas par une moindre efficacité des hôpitaux publics.

\section{Abstract \\ How to compare productivity and efficiency of public and private hospitals ?}

Brigitte Dormont (University Paris Dauphine) and Carine Milcent (PSE)

There is ongoing debate about the effect of ownership on hospital performance as regards efficiency and care quality. This paper proposes an analysis of the differences in productivity and efficiency between French public and private hospitals. In France, public and private hospitals do not only differ in their objectives. They are also subject to different rules as regards investments and human resources management. In addition, they were financed according to different payment schemes until 2004: a global budget system was used for public hospitals, while private hospitals were paid on a fee-for-service basis.

Since 2004, a prospective payment system (PPS) with fixed payment per stay in a given DRG is gradually introduced for both private and public hospitals. Payments generally differ for the same DRG, depending on whether the stay occurred in a private or public hospital. By 2018, a convergence of payments between the private and public sector should be achieved. Pursuing such a convergence comes down to suppose that there are differences in efficiency between 
private and public hospitals, which would be reduced by the introduction of competition between these two sectors.

The purpose of this paper is to compare the productivity of public and private hospitals in France. We try to assess the respective impacts, on productivity differences, of differences in efficiency, patient characteristics and production composition. We have chosen to estimate a production function. For that purpose, we have defined a variable measuring the volume of care services provided by each hospital, synthetizing the hospital multiproduct activity into one homogenous output.

Our data comes from two administrative sources which record exhaustive information about French hospitals. Matching these two database provides us an original source of information, at the hospital-year level, about both the production composition (number of stays in each DRG), and production factors (number of beds, facilities, number of doctors, nurses, of administrative and support staff, etc.). We observe 1,604 hospitals over the period 1998-2003, of which 642 hospitals are public, 126 are private not-for-profit and 836 are private-for-profit. This database is relative to acute care and covers more than $95 \%$ of French hospitals.

We use a stochastic production frontier approach combined with hospitals fixed effects. We find that the lower productivity of public hospitals is not explained by inefficiency (distance to the frontier), but oversized establishments, patient characteristics and production characteristics (small proportion of surgical stays). Once patient and production characteristics are taken into account, large and medium sized public hospitals appear to be more efficient than private hospitals. As a result, payment convergence would provide incentives for public hospitals to change the composition of their supply for care.

\section{Introduction}

En France les cliniques privées jouent un rôle important dans l'offre de soins hospitaliers. En 2007, $56 \%$ des séjours ont eu lieu dans des hôpitaux publics, 8 \% dans des hôpitaux privés à but non lucratifs, qui participent au service public hospitalier (PSPH) et $36 \%$ dans des cliniques privées, c'està-dire des hôpitaux privés à but lucratif ${ }^{2}$ (Arnault et al., 2009). Plusieurs rapports administratifs ont récemment montré qu'un séjour dans un hôpital public ou PSPH était plus coûteux que dans une clinique privée, suggérant que la productivité du secteur public était relativement faible (Aballea et al., 2006; DHOS, 2009). Les défenseurs du service public hospitalier soutiennent que cette différence de productivité n'est pas due à un défaut d'efficience mais résulte des obligations auxquelles sont

\footnotetext{
${ }^{2}$ On distingue habituellement les soins aigus (appelés aussi de court séjour, ou encore de médecine, chirurgie obstétrique - $\mathrm{MCO}$ ) des autres soins (soins de longue durée, soins de suite et de réadaptation ou soins psychiatriques). Pour les soins aigus on repère des séjours en hospitalisation complète (au moins une nuit passée à l'hôpital) et partielle (venues de moins d'un jour). Nous donnons ici les proportions correspondant à notre champ d'étude : les séjours pour soins aigus en hospitalisations complètes et partielles.
} 
soumis les hôpitaux publics et PSPH. En effet, ils ne peuvent pas sélectionner leurs patients et doivent organiser leur offre de soins en rapport avec des considérations d'intérêt général, ce qui limite les stratégies de spécialisation dans des activités lucratives.

Cet article a pour but de comprendre les différences de productivité observées en France entre les hôpitaux publics, les hôpitaux PSPH et les cliniques privées. Plus précisément, nous cherchons à évaluer le rôle, dans l'explication des différences de productivité, des différences d'efficacité productive et des différences dans les caractéristiques des patients et la composition de la production.

\section{Performance et statut des hôpitaux : que sait-on ?}

Les hôpitaux publics sont-ils nécessairement moins efficaces ? De nombreuses études cherchent à comprendre l'impact du statut de l'hôpital sur ses performances. Théoriquement, les différences de statut vont de pair avec des différences d'objectifs, avec pour conséquence des performances inégales. En principe, les hôpitaux publics sont peu incités à fonctionner de façon efficace et les hôpitaux privés à but non lucratif tendent à offrir une qualité et une quantité de services dépassant le niveau socialement optimal (Newhouse,1970, Lakdawalla et Philipson, 2006). Logiquement, les hôpitaux privés à but lucratif doivent être les plus efficaces (en termes de coût de traitement d'une affection donnée): ils cherchent à maximiser leur profit et peuvent minimiser les dimensions non contractualisables de la qualité (Hart et al., 1997). De façon générale, les différences de performance entre statuts doivent être réduites avec un système de tarification comme la T2A, qui se réfère à un mécanisme de concurrence par comparaison (encadré A).

Les études empiriques disponibles examinent l'impact du statut sur l'efficacité productive, les comportements de demande induite et la qualité des soins.

En ce qui concerne l'efficacité, les études américaines n'aboutissent pas à des conclusions définitives : d'après Sloan (2000) il n'y a pas de différence systématique entre les hôpitaux privés à but lucratif et non lucratif. Burgess et Wilson (1996) soulignent que le caractère multiproduit de l'activité hospitalière conduit à définir l'efficacité sur plusieurs dimensions. Pour les Etats-Unis, ils trouvent que les hôpitaux publics de la "Veteran Administration " (destinés aux militaires) sont plus efficaces dans certaines dimensions que les hôpitaux privés à but lucratif et non lucratif, mais que leur taille excessive les rend très inefficaces du point de vue de leur échelle de production (scale inefficiency). Sur données allemandes, Herr et al. (2010) montrent que les hôpitaux privés à but lucratif ne sont pas plus efficaces du point de vue de leurs coûts que les hôpitaux publics mais qu'ils ont une meilleure rentabilité.

Les autres travaux empiriques examinent si le statut de l'hôpital peut influencer le coût et la qualité du traitement pour un patient admis avec une maladie donnée. Si les soins hospitaliers sont rémunérés sur la base d'un paiement à l'acte, ce vers quoi peut facilement dériver la tarification à l'activité (qui porte bien son nom), les hôpitaux privés à but lucratif ont intérêt à pratiquer des procédures plus intensives et en plus grand nombre. Aux Etats-Unis aussi, la tarification par pathologie est configurée de telle sorte qu'un traitement plus intensif conduit à un classement du séjour dans un groupe plus rémunérateur. Plusieurs études sur ce pays montrent que les patients 
affectés d'un problème de santé donné (crise cardiaque, fracture de la hanche, accident vasculaire cérébral) ont des traitements plus coûteux s'ils sont admis dans un hôpital privé à but lucratif (Sloan et al., 2001). En revanche ce type de résultats n'est pas confirmé pour les hôpitaux de Taïwan (Lien et al., 2008). Lorsqu'ils existent, les comportements de demande induite conduisent à s'écarter de l'efficacité allocative et génèrent des dépenses excessives. Ils montrent que la question de l'efficacité productive des hôpitaux ne se réduit pas à la minimisation du coût pour un traitement donné.

Les travaux empiriques qui s'intéressent à la qualité montrent que les hôpitaux privés à but lucratif délivrent des soins de moindre qualité que les autres hôpitaux. La privatisation d'un hôpital conduit aussi à une baisse de qualité (Picone et al., 2002; Lien et al., 2008). Ces résultats concernent d'autres pays que la France: ils comparent principalement la qualité des soins des hôpitaux privés à but lucratif avec celle des hôpitaux privés à but non lucratif, sans beaucoup examiner le cas des hôpitaux publics. Pour la France, en considérant le traitement de l'infarctus du myocarde, Milcent (2006) montre que le taux de mortalité durant le séjour est en moyenne supérieur dans les établissements privés, avec cependant une plus grande hétérogénéité des taux de mortalité observés dans les cliniques par rapport à ce qui est observé dans les hôpitaux publics. Le rapport s'inverse lorsque l'on tient compte de la mise en œuvre des actes innovants : Gobillon et Milcent (2010) trouvent alors que le taux de mortalité durant le séjour est inférieur dans les hôpitaux publics.

Notre étude examine l'efficacité productive au sens restreint du terme, sans se pencher sur les questions de demande induite. Autrement dit, nous n'étudions pas la relation entre problème de santé à l'admission du patient, décision de traitement et coût final du séjour. En mobilisant des données de séjours hospitaliers, nous étudions l'impact du statut des hôpitaux sur leur productivité et leur efficacité technique, en prenant comme donné le nombre observé de séjours dans chaque groupe homogène de malades (GHM).

\section{Le débat français}

En France comme dans les autres pays, l'hôpital coûte très cher. En 2009, les soins hospitaliers représentent $44,4 \%$ de la consommation de soins et de biens médicaux, soit le premier poste de dépense (DREES, 2011), alors que les séjours hospitaliers ne concernent chaque année que $10 \%$ de la population environ. Plus précisément, les soins dans les hôpitaux publics et PSPH représentent $34,1 \%$ de la consommation de soins et de biens médicaux et les cliniques privées $10,3 \%$.

L'introduction de la Tarification à l'Activité (T2A) en 2004 visait à améliorer l'efficacité de la dépense pour ce poste majeur que constituent les soins hospitaliers. La mise en œuvre du nouveau paiement est progressive, avec une application intégrale à partir de 2008 (encadré A). Dès le départ, les tarifs diffèrent pour un même Groupe Homogène de Malades (GHM), selon que le séjour a eu lieu dans un hôpital public (ou PSPH) ou un hôpital privé à but lucratif ${ }^{3}$. Actuellement, les paiements par séjour dans un GHM donné sont en moyenne 27 \% plus élevés dans le secteur public (et PSPH) que dans le

\footnotetext{
${ }^{3}$ La terminologie employée par l'administration pour décrire cette situation distingue GHM et GHS (groupes homogènes de séjours). Selon la Mission T2A, « Si le principe de base repose sur une seule classification en GHM pour l'ensemble des secteurs MCO, 2 barèmes distincts coexistent (soit 2 échelles de GHS) ". Dans cette terminologie, le GHS désigne le tarif par séjour dans un GHM donné.
} 
secteur privé (DHOS, 2009). Les discussions sur l'évaluation des écarts de coûts public-privé sont innombrables : l'écart s'est rapidement réduit, de 80 \% environ en 2006 (Aballea et al., 2006) aux $27 \%$ susmentionnés, sans que l'on comprenne bien pourquoi. L'hypothèse est parfois émise, d'une prise en charge partielle du coût des séjours par un développement incontrôlé des enveloppes forfaitaires attribuées majoritairement aux hôpitaux publics pour les missions d'intérêt général qu'ils assument (voir encadré $A$ ).

Une convergence des grilles tarifaires des secteurs public et privé, d'abord programmée pour 2012, est maintenant prévue à l'horizon 2018. Mettre en place cette convergence revient à supposer que les différences de coûts sont exclusivement dues à des différences d'efficacité, qui seraient éliminées par l'introduction d'une concurrence entre les deux secteurs. Les cliniques privées développent actuellement un fort lobbying en faveur de la convergence tarifaire, laquelle pourrait leur conférer d'importantes rentes à court terme, compte tenu de leurs plus faibles coûts de traitement dans nombre de GHM. Au contraire, les défenseurs des hôpitaux publics et PSPH s'opposent à une telle réforme, en invoquant leurs missions de service public pour expliquer les différences de coût.

En pratique, une tarification comme la T2A conduit à des budgets qui sont des fonctions linéaires du nombre de séjours dans chaque GHM. L'hypothèse implicite d'un tel calcul est qu'il n'y a pas d'économie d'échelle ni d'économie de gamme. Certes, l'organisation actuelle prévoit que les hôpitaux peuvent recevoir des enveloppes budgétaires additionnelles pour des activités comme l'enseignement, la recherche, les soins palliatifs, la gériatrie, les urgences et la prise en charge des populations précaires (encadré A). Mais, dans le champ de la T2A, le paiement des séjours en soins aigus est une formule linéaire qui n'intègre pas d'éventuelles économies liées au nombre des séjours ou à leur composition.

Notre objectif est précisément d'examiner s'il existe une influence de la composition des séjours sur la productivité des hôpitaux en matière de soins aigus. Si tel est le cas, introduire de la concurrence entre les établissements sur la base de la T2A crée de fortes pressions en faveur d'une réorganisation de l'offre de soins, qui s'ajoutent aux encouragements aux restructurations hospitalières déjà existant. Ces changements peuvent être des fusions ou des fermetures d'établissements, largement observées aux Etats-Unis, des changements de statut (privatisation) ou plus simplement des changements dans la structure des séjours à l'intérieur de chaque hôpital, comme par exemple une augmentation de la proportion de séjours chirurgicaux. Ces changements sont souhaitables s'ils permettent d'améliorer l'efficacité dans la délivrance des soins hospitaliers. En revanche, il n'est pas souhaitable que le système de tarification crée des incitations à la sélection de patients ou à l'arrêt de la production de soins qui seraient importants du point de vue du bien-être collectif. Afin que les besoins de la population soient satisfaits, les autorités administratives imposent aux hôpitaux publics un cahier des charges que ne partagent pas les cliniques privées. La tarification hospitalière doit-elle tenir compte des différences dans la composition de l'activité ? Cette question est d'une importance primordiale, dès lors que l'on met en concurrence des hôpitaux soumis à des règles différentes.

\section{En France les hôpitaux ne sont pas soumis aux mêmes contraintes selon leur statut}

Les hôpitaux publics, privés et PSPH sont soumis à des règles différentes concernant leurs investissements, la gestion des ressources humaines et la sélection des patients. Dans le secteur public, les médecins, infirmier(e)s et autres personnels sont fonctionnaires, ce qui limite la flexibilité dans les ajustements d'effectifs et la définition des rémunérations. Par ailleurs, les hôpitaux publics 
doivent garantir à tous les patients potentiels un accès continu aux soins, vingt-quatre heures sur vingt-quatre ${ }^{4}$. Dans le secteur privé à but lucratif, en revanche, les décisions dépendent principalement de la demande qui s'adresse à l'hôpital. Celui-ci peut se spécialiser dans tel ou tel segment des soins hospitaliers, en fonction des conditions prévalant sur le marché des soins. Les employés salariés ont des contrats de droit privé, et la plupart des médecins qui travaillent dans les cliniques sont des libéraux payés par honoraires, ce qui offre aux établissements une extrême souplesse dans la gestion des séquences et des durées d'intervention. Dernier avantage, et non des moindres, les hôpitaux à but lucratif peuvent sélectionner leurs patients. Moins nombreux, les hôpitaux privés à but non lucratif (PSPH) constituent une sorte de catégorie intermédiaire. Ils sont soumis à des contraintes proches de celles des hôpitaux publics, sauf en ce qui concerne la gestion des ressources humaines : les contrats de travail sont de droit privé et les rémunérations ne sont pas soumises à la grille de la fonction publique.

Les hôpitaux publics sont-ils nécessairement moins productifs que les hôpitaux privés ? Leurs missions diffèrent, ainsi que les règles relatives à la gestion des ressources humaines et la sélection de patients. Leurs objectifs diffèrent aussi, qui découlent des différences de statut. En pratique, les hôpitaux privés à but lucratif sont fortement incités par leurs actionnaires ou propriétaires à générer un profit. A l'inverse, les hôpitaux publics n'ont pas de raison de chercher à réaliser un excédent budgétaire, ni à le maximiser. Jusqu'à l'introduction de la T2A, le fonctionnement de la tutelle administrative avait même pour effet de décourager toute recherche d'efficacité : en général, un surplus dégagé une année $t$ était récupéré par la tutelle et une somme équivalente soustraite du budget de l'année suivante $(t+1)$. Dans un tel contexte, les établissements du service public avaient intérêt à afficher une situation d'équilibre budgétaire, voire de déficit.

Des systèmes de paiement différents ont-ils pu favoriser d'inégales performances en matière d'efficacité productive ? Jusqu'en 2003, les hôpitaux publics et PSPH étaient financés par un budget global, alors que les cliniques privées étaient financées par un système combinant paiement à l'acte et prix de journée pour couvrir les frais d'hébergement. En pratique, les cliniques étaient donc financées par un paiement de nature rétrospective. Dans le secteur public, la contrainte budgétaire associée au budget global était plutôt "douce »: aucun déficit ne menaçait sérieusement la pérennité d'un établissement, ce qui confère au budget global aussi un caractère plutôt rétrospectif. Les systèmes de paiement en vigueur étaient donc de nature rétrospective pour tous les hôpitaux, qu'ils soient publics, PSPH ou privés, ce qui n'incite guère à l'efficacité (encadré $A$ ).

Quelles sont les caractéristiques des hôpitaux privés à but lucratif en France ? Ils occupent une place importante dans l'offre de soins hospitaliers : un tiers des séjours pour soins aigus ont lieu dans une clinique privée ${ }^{5}$. On observe une forte spécialisation en séjours ambulatoire (de moins de vingtquatre heures) et chirurgicaux : actuellement, la moitié des séjours chirurgicaux ont lieu dans des cliniques privées. A l'origine, les cliniques étaient possédées et gérées par un médecin ou un groupe de médecins. Maintenant que cette génération de médecins arrive à l'âge de la retraite, le rachat de leurs établissements par des fonds de pension ou des fonds d'investissement conduit à la

\footnotetext{
${ }^{4}$ Pour des tâches transversales correspondant à des missions d'intérêt général (enseignement et recherche, Samu, etc.) les hôpitaux publics reçoivent des paiements annexes dans le cadre de la T2A (voir encadré A).

${ }^{5}$ Aux Etats-Unis la part du privé à but lucratif est très inférieure. En Allemagne, elle était de $15 \%$ en 1991, pour atteindre $30 \%$ en 2007, au terme d'une forte vague de privatisation (Herr et al., 2010).
} 
constitution de grandes chaînes de cliniques, comme par exemple La Générale de Santé, ou Vitalia. Ces investissements témoignent de la rentabilité potentielle des soins hospitaliers privés.

\section{Définition de la production des hôpitaux}

En matière de soins aigus, un hôpital peut offrir des soins très variés, avec des séjours pour accouchement, pour prendre en charge une crise cardiaque, mettre en place une prothèse de hanche, opérer une cataracte, etc. Ainsi, l'hôpital peut être considéré comme une entreprise multiproduit, chaque produit correspondant aux séjours dans les différents GHM. Dans ce cadre, I'approche la plus directe consiste à considérer une fonction de coût. Ce type de spécification permet en effet d'étudier assez simplement les activités multiproduit.

Mais les données disponibles ne permettent pas de mener une comparaison fiable des secteurs public et privé de l'hospitalisation sur la base des coûts. En effet, ces derniers ne sont connus que pour un sous-échantillon assez restreint d'établissements dans le secteur public et sont très difficiles à observer dans le secteur privé à but lucratif. L'information sur les coûts des cliniques est plutôt "sensible", compte tenu de l'environnement concurrentiel auquel elles sont exposées. Par ailleurs, des médecins sont souvent partiellement propriétaires d'une clinique et obtiennent une partie de leur rémunération sous la forme d'une participation au résultat, ce qui crée une difficulté à mesurer les vrais coûts et la rentabilité de l'établissement. Enfin, le périmètre du coût varie selon les secteurs : il n'inclut pas les rémunérations des médecins libéraux dans le secteur privé. Force a été d'abandonner l'approche en termes de fonction de coût et de considérer une fonction de production.

Dans ce but, nous avons synthétisé l'activité "multiproduit" de l'hôpital par un produit homogène défini selon des critères identiques, quel que soit le statut de l'hôpital :

$$
Q_{h t}=\sum_{j=1}^{J} p_{j t} N_{j h t}
$$

où $N_{j h t}$ désigne le nombre de séjours réalisés par l'hôpital $h$ pour le GHM $j$ à l'année $t$.

$p_{j t}, j=1, \ldots, J ; t=1, \ldots, T$, est le nombre de points ISA (Indice synthétique d'activité, voir encadré A) associé pour l'année $t$ à un séjour dans le GHM $j$. II s'agit de l'échelle de poids utilisée par l'administration pour calculer la production des hôpitaux publics ou PSPH en nombres de points ISA. Ces poids sont basés sur les coûts relatifs des séjours par GHM, estimés à partir du sous-échantillon d'établissements du secteur public pour lesquels les coûts sont observés.

Point fondamental : nous utilisons la même échelle de poids - celle du secteur public - pour valoriser la production des hôpitaux publics, PSPH et des cliniques privées. En effet, une échelle unique doit être utilisée pour une comparaison pertinente de la productivité dans les différents secteurs. De façon symétrique, il aurait été intéressant d'utiliser aussi, pour valoriser les séjours des hôpitaux publics, PSPH et privés, l'échelle des coûts du secteur privé à but lucratif. Mais cette échelle n'est pas disponible pour la période d'étude. 


\section{Productivité et organisation des hôpitaux publics et privés : éléments d'analyse descriptive}

Nos données sont constituées à partir de deux sources administratives : les données du Programme de médicalisation des systèmes d'information (PMSI) et celles de la Statistique Annuelle des Etablissements de Santé (SAE). Nous observons 1604 hôpitaux sur la période 1998-2003, avec des informations au niveau hôpital-année sur les soins délivrés et les facteurs de production mobilisés (encadré B). Le champ d'étude est celui des courts séjours en médecine, chirurgie obstétrique ${ }^{6}-$ MCO - en hospitalisations complètes (au moins une nuit passée à l'hôpital) et partielles (venues de moins d'un jour).

Notre analyse couvre les six années précédant l'introduction de la T2A en France afin d'observer précisément la situation qui préexistait avant la mise en place de nouvelles incitations. Ce travail permet d'avoir un référentiel sur la situation du tissu hospitalier français et les performances comparées des établissements publics, PSPH et privés, avant la mise en place de la réforme. En effet, les incitations introduites par la T2A entraînent nécessairement des réactions stratégiques des comportements des acteurs. II est important d'évaluer l'impact du statut de l'hôpital sur sa productivité et son efficacité avant l'introduction de ces mécanismes. Outre l'intérêt intrinsèque de cette question, ceci permettra, dans un deuxième temps, d'évaluer rigoureusement l'impact de la réforme sur les performances comparées des hôpitaux.

\footnotetext{
${ }^{6}$ Par opposition aux soins de longue durée, aux soins de suite et de réadaptation ou aux soins psychiatriques.
} 
Tableau 1 : Caractéristiques des données

\begin{tabular}{|c|c|c|c|c|c|c|c|c|c|}
\hline Taille & Statut & $\begin{array}{c}\text { Nombre } \\
\text { d'hôpitaux }\end{array}$ & $\begin{array}{l}\text { Nombre de } \\
\text { lits par } \\
\text { hôpital }\end{array}$ & $\begin{array}{c}\text { Nombre } \\
\text { annuel de } \\
\text { séjours par } \\
\text { hôpital } \\
\end{array}$ & $\begin{array}{c}\text { Proportion (\%) } \\
\text { dans la production } \\
\text { totale } \\
\text { [dans le nombre } \\
\text { total de séjours] } \\
\end{array}$ & $\begin{array}{c}\text { Durée de séjour } \\
\text { (DS) moyenne * } \\
\text { [moyenne de la } \\
\text { DS médiane] }\end{array}$ & $\begin{array}{l}\text { Nombre de } \\
\text { séjours par lit }\end{array}$ & $\begin{array}{c}\text { Proportion } \\
(\%) \text { de } \\
\text { séjours } \\
\text { chirurgicaux } \\
\end{array}$ & $\begin{array}{l}\text { Production } \\
\text { annuelle par } \\
\text { lit ** }\end{array}$ \\
\hline \multirow{3}{*}{ Petit } & Public & 282 & 45 & 1794 & $\begin{array}{c}2.7 \\
{[3.0]}\end{array}$ & $\begin{array}{c}9.3 \\
{[7.1]}\end{array}$ & 33 & 7.9 & 43225 \\
\hline & PSPH & 72 & 64 & 2499 & $\begin{array}{c}1.3 \\
{[1.1]}\end{array}$ & $\begin{array}{c}6.9 \\
{[4.6]}\end{array}$ & 37 & 20.9 & 62405 \\
\hline & Privé & 541 & 58 & 2986 & $\begin{array}{c}11.1 \\
{[11.7]}\end{array}$ & $\begin{array}{c}3.9 \\
{[2.3]}\end{array}$ & 54 & 47.2 & 73785 \\
\hline \multirow{3}{*}{ Moyen } & Public & 117 & 151 & 7129 & $\begin{array}{c}6.0 \\
{[6.9]}\end{array}$ & $\begin{array}{c}5.4 \\
{[3.5]}\end{array}$ & 48 & 20.5 & 60222 \\
\hline & PSPH & 40 & 153 & 6811 & $\begin{array}{c}2.5 \\
{[2.0]}\end{array}$ & $\begin{array}{c}4.5 \\
{[2.5]}\end{array}$ & 45 & 29.8 & 81914 \\
\hline & Privé & 234 & 118 & 6823 & $\begin{array}{c}14.3 \\
{[14.1]}\end{array}$ & $\begin{array}{c}3.5 \\
{[1.8]}\end{array}$ & 60 & 50.4 & 87201 \\
\hline \multirow{3}{*}{ Grand } & Public & 243 & 566 & 26865 & $\begin{array}{c}53.4 \\
{[53.0]}\end{array}$ & $\begin{array}{c}5.3 \\
{[2.7]}\end{array}$ & 49 & 18.0 & 67390 \\
\hline & PSPH & 14 & 339 & 15303 & $\begin{array}{c}1.6 \\
{[1.4]}\end{array}$ & $\begin{array}{c}4.7 \\
{[2.4]}\end{array}$ & 47 & 24.8 & 78604 \\
\hline & Privé & 61 & 201 & 12381 & $\begin{array}{c}7.3 \\
{[6.7]}\end{array}$ & $\begin{array}{c}3.8 \\
{[2.1]}\end{array}$ & 63 & 44.6 & 97242 \\
\hline Total & & $\begin{array}{c}1604 \\
\text { (7 731 obs) }\end{array}$ & 169 & 8334 & $\begin{array}{c}100.0 \\
{[100.0]}\end{array}$ & $\begin{array}{c}5.1 \\
{[3.1]}\end{array}$ & 50 & 33.5 & 70517 \\
\hline
\end{tabular}

1604 hôpitaux 1998-2003, 7731 observations dans la dimension hôpital-année

"La durée de séjour (DS) est mesurée en jours: nous donnons la moyenne de la moyenne de la DS calculée pour chaque hôpital et, entre crochets, la moyenne de la médiane de la DS calculée pour

chaque hôpital. "La production est mesurée par le nombre de points ISA (en milliers) 
Graphique 1

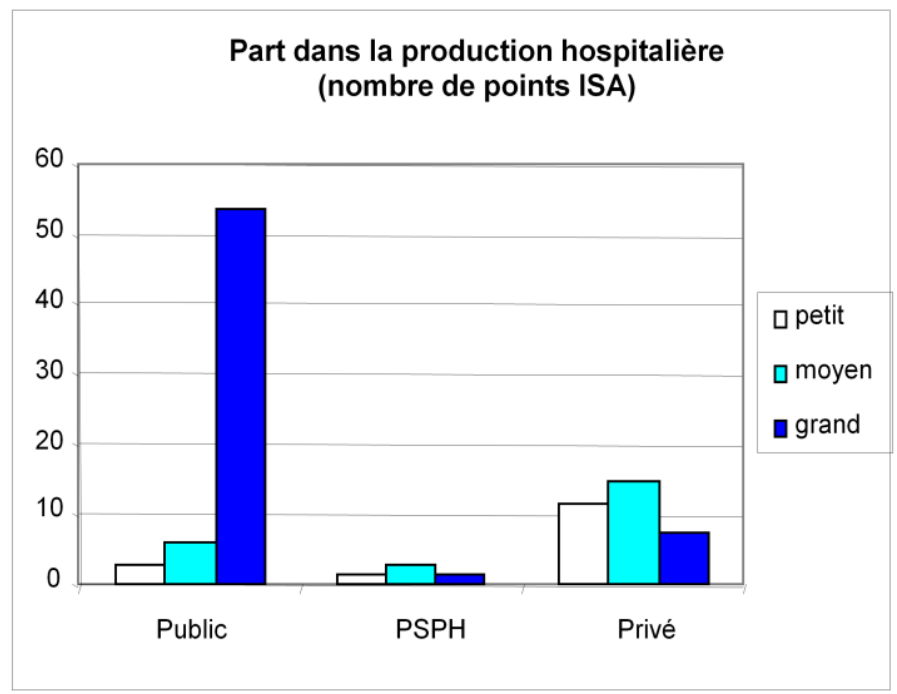

Graphique 2

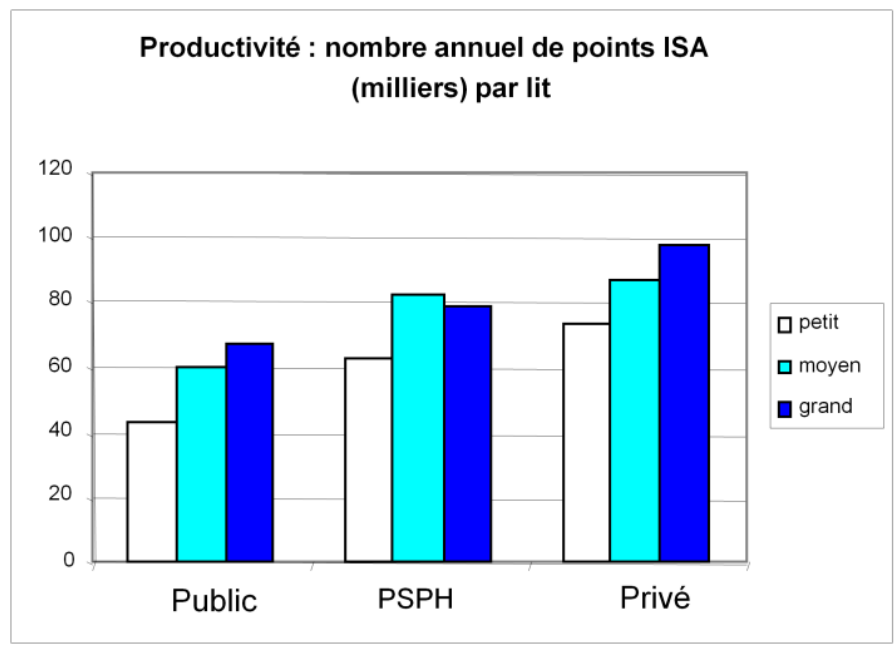

Graphique 3

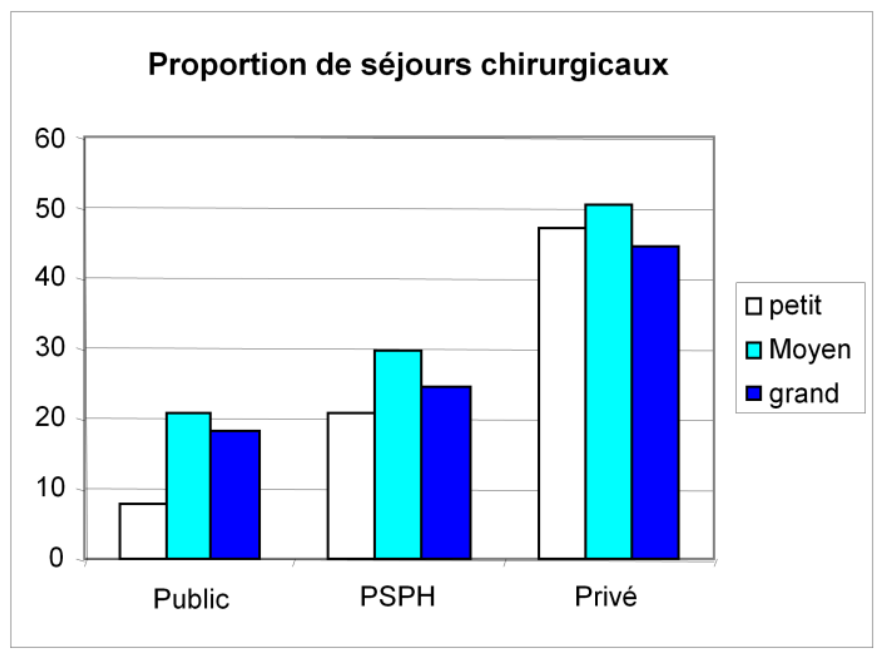


Sur les 1604 hôpitaux observés, 642 sont publics, 126 sont des hôpitaux PSPH et 836 des cliniques privées. Nous avons introduit une distinction selon la taille de l'établissement en adoptant le critère du nombre de séjours proposé par l'Agence Technique de I'Information sur l'Hospitalisation (ATIH) : les hôpitaux sont " petits ", " moyens » ou " grands " selon qu'ils ont eu moins de 5000 , entre 5000 et 10000 ou plus de 10000 séjours dans l'année.

Les hôpitaux publics assument la plus grande part des soins hospitaliers : $62,9 \%$ des séjours ont lieu dans un hôpital public, 4,6\% dans un hôpital PSPH et 32,5\% dans une clinique. Des proportions comparables sont obtenues si l'on mesure les services hospitaliers par le nombre de points ISA (tableau 1). Le graphique 1 montre ce sont les grands hôpitaux publics qui délivrent la majeure partie des soins (plus de $50 \%$ ). Les cliniques privées délivrent une quantité de soins plus modeste, mais non négligeable quelle que soit leur taille ( $11 \%$ et $14 \%$ pour les petites et moyennes cliniques). En mesurant la productivité par le nombre de points ISA par lit, on observe que les hôpitaux publics sont moins productifs que les hôpitaux PSPH et les cliniques, et ce quelle que soit leur taille (graphique 2). Ceci peut être rapproché du fait que l'activité des cliniques privées se caractérise par une très importante proportion de séjours chirurgicaux, qui approche les 50 \% (graphique 3).

Le tableau 1 présente d'autres détails sur les caractéristiques des hôpitaux, telles qu'elles apparaissent dans notre base de données (proche de l'exhaustif). Dans la catégorie des établissements qui réalisent moins de 5000 séjours annuels, on observe que les cliniques sont presque deux fois plus nombreuses que les hôpitaux publics, avec plus de lits ( 58 versus 45 ). Dans la catégorie des établissements qui réalisent entre 5000 et 10000 séjours annuels, les cliniques sont encore deux fois plus nombreuses, mais ont cette fois-ci moins de lits que les hôpitaux publics (118 versus 151). Les hôpitaux publics dominent la catégorie des établissements à forte activité (plus de 10000 séjours annuels) : dans cette catégorie 243 hôpitaux sont publics contre seulement 14 PSPH et 61 cliniques privées. Ces hôpitaux publics ont un nombre de lits considérable : 566 lits en moyenne alors que les cliniques de la même catégorie n'ont que 201 lits.

Les hôpitaux publics se caractérisent par une faible proportion de séjours chirurgicaux mais aussi par la longueur des séjours. Ceci est particulièrement marqué pour les petits hôpitaux publics, dans lesquels la durée de séjour est en moyenne de 9,3 jours alors qu'elle est de 3,8 jours dans les petites cliniques. L'écart entre les durées de séjours des hôpitaux publics et privés se réduit mais reste non négligeable (de 1,5 à 2 jours) pour des structures hospitalières de taille supérieure. Concernant la part des séjours chirurgicaux et la durée des séjours, les caractéristiques des hôpitaux PSPH se situent entre celles des hôpitaux publics et des cliniques privées (tableau 1).

Nos données offrent aussi des informations sur les facteurs de production, notamment sur le nombre de lits et la composition du personnel (tableau 2). En moyenne, les établissements emploient 3 personnes par lit. Mais ce chiffre recouvre de fortes disparités selon la taille et le statut de l'hôpital. Le nombre d'employés par lit passe de 7,6 personnes pour les petits hôpitaux publics à 3,7 pour les hôpitaux publics moyens ou grands. Par ailleurs, le nombre de personnes par lit est toujours beaucoup plus faible dans les cliniques : quelle que soit leur taille, elles emploient entre 1,7 et 1,9 personnes par lit. 
Tableau 2 : Composition du personnel au sein de l'hôpital

\begin{tabular}{|c|c|c|c|c|c|c|c|c|}
\hline Taille & Statut & $\begin{array}{l}\text { Nombre } \\
\text { de lits }\end{array}$ & $\begin{array}{c}\text { Nombre de } \\
\text { personnes } \\
\text { par lit }\end{array}$ & $\begin{array}{l}\text { Médecins }^{\#} \\
\text { par lit }\end{array}$ & $\begin{array}{c}\text { Infirmiers } \\
\text { par lit }\end{array}$ & $\begin{array}{c}\text { Aide- } \\
\text { soignants } \\
\text { par lit } \\
\end{array}$ & $\begin{array}{c}\text { Administr. } \\
\text { par lit }\end{array}$ & $\begin{array}{l}\text { Médico- } \\
\text { technique } \\
\text { par lit } \\
\end{array}$ \\
\hline \multirow{3}{*}{ Petit } & Public & $45^{* * *}$ & $7.62^{* * *}$ & $0.24^{* * *}$ & $1.63^{* * *}$ & $3.84^{* * *}$ & $0.68^{* * *}$ & $1.23^{* * *}$ \\
\hline & PSPH & $64^{* * * *}$ & 3.56 & $\begin{array}{c}0.20^{* * *} \\
{[0.15 ; 0.24]}\end{array}$ & 1.10 & 1.12 & $0.53^{* * *}$ & 0.62 \\
\hline & Privé & $58^{* * *}$ & $1.76^{* * *}$ & $\begin{array}{c}0.26^{* * *} \\
{[0.13 ; 0.36]}\end{array}$ & $0.51^{* * * *}$ & $0.59^{* * *}$ & $0.25^{* * *}$ & $0.14^{* * *}$ \\
\hline \multirow{3}{*}{ Moyen } & Public & $151^{* * *}$ & 3.66 & $0.29^{* *}$ & $1.08^{* *}$ & $1.33^{* * *}$ & 0.38 & $0.57^{* * *}$ \\
\hline & PSPH & $153^{* * *}$ & $2.62^{* * *}$ & $\begin{array}{c}0.17^{* * * *} \\
{[0.15 ; 0.19]}\end{array}$ & $0.83^{* * *}$ & $0.71^{* * *}$ & $0.44^{* *}$ & $0.47^{* * *}$ \\
\hline & Privé & $118^{* * *}$ & $1.67^{* * *}$ & $\begin{array}{c}0.22^{* * *} \\
{[0.13 ; 0.29]}\end{array}$ & $0.54^{* * * *}$ & $0.58^{* * * *}$ & $0.21^{* * *}$ & $0.12^{* * *}$ \\
\hline \multirow{3}{*}{ Grand } & Public & $566^{(\mathrm{ref})}$ & $3.65^{\text {(ref) }}$ & $0.32^{\text {(ref) }}$ & $1.16^{(\mathrm{ref})}$ & $1.15^{\text {(ref) }}$ & $0.39^{\text {(ref) }}$ & $0.63^{\text {(ref) }}$ \\
\hline & PSPH & $339^{* * *}$ & $2.86^{* * *}$ & $\begin{array}{c}0.13^{* * *} \\
{[0.11 ; 0.14]}\end{array}$ & $0.95^{* *}$ & $0.77^{*}$ & $0.47^{* *}$ & 0.55 \\
\hline & Privé & $201^{* * *}$ & $1.91^{* * *}$ & $\begin{array}{c}0.27^{* * *} \\
{[0.17 ; 0.35]}\end{array}$ & $0.63^{* * * *}$ & $0.65^{* * *}$ & $0.21^{* * *}$ & $0.14^{* * *}$ \\
\hline $\begin{array}{l}\text { Moyenne } \\
\text { générale }\end{array}$ & & 169 & 3.14 & 0.26 & 0.87 & 1.23 & 0.35 & 0.44 \\
\hline
\end{tabular}

1604 hôpitaux 1998-2003, 7731 observations

La différence avec le niveau moyen dans les grands hôpitaux est significatif $1 \%\left(^{* * *}\right), 5 \%\left(\left(^{* *}\right), 10 \%\left(^{*}\right)\right.$

\# Les médecins sont observés sur un échantillon de 1169 hôpitaux seulement (5 798 observations sur 1998-2003). Pour les hôpitaux privés et PSPH on suppose que les médecins à temps partiel travaillent à mi- temps (coefficient 0.5 ). Entre parenthèses figurent les valeurs obtenues avec d'autres hypothèses sur le taux permettant de calculer l'équivalent temps plein des médecins à temps partiel : coefficients 0.1 and 0.8 . 
Ce constat est maintenu lorsque l'on étudie le personnel à un niveau plus détaillé : par rapport aux cliniques, les hôpitaux publics emploient plus d'infirmièr(e)s par lit, d'aides-soignant(e)s, de personnel administratif et de personnel médico-technique par lit. Le contraste est marqué pour les aides-soignant(e)s par lit dans les petits établissements : ce ratio est plus de six fois plus grand dans le public que dans le privé. Surtout, le personnel médico-technique par lit est particulièrement important dans les hôpitaux publics : quelle que soit leur taille, le nombre de personnes par lit de cette catégorie y est de 4 à 8 fois plus élevé que dans les cliniques. A l'instar de ce qui était observé sur les durées de séjour, la structure du personnel hospitalier des établissements PSPH a des caractéristiques qui apparaissent comme intermédiaires, situées entre celles des hôpitaux publics et celles des cliniques.

Nous publions aussi des statistiques sur le nombre de médecin par lit. Les chiffres obtenus doivent être interprétés avec précaution : ils sont calculés à partir d'un sous-échantillon de 1169 hôpitaux pour lesquels le nombre de médecins est renseigné. L'information sur les durées d'activité n'est pas disponible, ce qui rend hasardeuse une évaluation en équivalent temps-plein des effectifs des médecins libéraux ou à temps partiel. Nous avons procédé à des évaluations en retenant trois hypothèses : temps partiel à $50 \%, 10 \%$ ou $80 \%$. A partir de ces mesures, on trouve que le nombre de médecins par lit en équivalent temps-plein diffère peu suivant le statut de l'hôpital (tableau 2). Cependant, nous n'avons aucun élément permettant de vérifier la pertinence des hypothèses envisagées; de surcroît, notre calcul revient à supposer que le coefficient de passage au temps-plein est homogène, quel que soit le statut et la taille de l'hôpital. Enfin, pour les cliniques, il est possible que la totalité des médecins libéraux ou certains d'entre eux ne soient pas recensés. Pour les estimations économétriques, il est plus raisonnable de traiter le nombre de médecins par lit comme une variable omise.

Au total, les établissements publics et PSPH emploient davantage de personnel par lit et ont des durées de séjour plus longues que les cliniques privées. Ceci suggère que les facteurs de production de soins hospitaliers sont utilisés de façon moins efficace dans le secteur public. Toutefois, ces caractéristiques peuvent aussi résulter de traits spécifiques de l'activité des hôpitaux publics, qui conduiraient à une importante proportion de séjours médicaux et à un besoin plus élevé de personnel.

\section{Efficacité productive des hôpitaux publics : le diagnostic dépend de la prise en compte des caractéristiques de leur activité}

Pour étudier l'efficacité productive des hôpitaux, nous estimons une fonction de production loglinéaire mettant en relation la productivité de l'hôpital et ses facteurs de production (encadré C). La productivité est définie, comme dans l'analyse descriptive, par le nombre de points ISA par lit et les facteurs de production sont le nombre d'infirmièr(e)s par lit, d'aides-soignant(e)s par lit, de personnel administratif par lit et de personnel médico-technique par lit. Pour les raisons données cidessus, il est préférable de traiter les médecins comme une variable omise, mais la robustesse des résultats à ce choix est contrôlée par une estimation annexe intégrant les observations relatives aux médecins, disponibles sur un échantillon restreint. 
Le fait de disposer de données de panel - avec 1604 hôpitaux observés sur les années 1998 à 2003permet d'estimer un terme $v_{h}$ pour l'hétérogénéité non observée propre à l'hôpital et un terme $u_{h} \geq 0$ mesurant son efficacité productive. Le terme relatif à l'hétérogénéité non observée capture l'impact d'éléments susceptibles d'influencer la productivité qui ne figurent pas dans la liste des variables explicatives: le nombre de médecins, la qualité des soins, le type de soins délivrés, la composition de l'activité, certaines caractéristiques des patients, etc. La fonction de production qui est spécifiée définit la frontière de production efficace : le nombre maximal de points ISA qui peuvent être obtenus avec une quantité donnée de facteurs de production utilisés. Le terme $u_{h}$ mesure la distance à cette frontière de production, distance attribuée à une utilisation inefficace des facteurs.

Bien évidemment, le contenu de l'hétérogénéité non observée $v_{h}$ varie avec la liste des variables explicatives retenues, dont l'extension revient à incorporer dans le modèle des variables omises. Pareillement, ajouter des variables explicatives à la spécification influence l'évaluation de l'efficacité $u_{h}$ car elle fait varier la localisation de la frontière.

Notre stratégie empirique consiste à partir d'une première spécification définie comme une fonction de production classique mettant en rapport inputs et output. Puis, à considérer des spécifications incorporant des variables explicatives additionnelles décrivant les caractéristiques des patients et de la production des hôpitaux. La fonction de production est supposée identique, quels que soient le statut et la taille de l'hôpital: telle est bien l'hypothèse d'un régulateur qui introduit une concurrence par comparaison entre tous les types d'établissements.

\section{La productivité est liée à des séjours simples avec une technologie intensive}

Quel que soit le modèle considéré, les estimations donnent des résultats similaires concernant l'influence des facteurs de production sur la productivité des hôpitaux (tableau 3). Les différentes catégories de personnel ont une productivité marginale positive, à l'exception des personnels médico techniques, dont le coefficient est négatif, ce qui suggère l'existence de sureffectifs (pour le moins !). Le coefficient - positif - des aides soignantes n'est significatif que dans le modèle III (qui intègre les caractéristiques des patients et des séjours dans ses variables explicatives), et seulement avec un risque de première espèce assez élevé, de $10 \%$. 
Tableau 3 : Estimation de la fonction de production (première étape) Variable dépendante: $\log$ (production par lit)

\begin{tabular}{|c|c|c|}
\hline & \multicolumn{2}{|c|}{ Modèle I Modèle II } \\
\hline $\log ($ lit $)$ & $-0,3318^{\star \star *}$ & $-0,4863^{\star \star *}$ \\
\hline Log (infirmière/ lit) & $0,2780^{* * *}$ & $0,1969^{* * *}$ \\
\hline Log (aide soignante/lit) & 0,0437 & $0,1060^{*}$ \\
\hline Log (administratif/lit) & $0,4562^{* * *}$ & $0,4092^{* \star *}$ \\
\hline Log (médico-technique/lit) & $-0,2973^{\star * *}$ & $-0,2562^{* * *}$ \\
\hline$\%$ femmes $19-40$ ans & & 0,2445 \\
\hline$\%$ hommes $19-40$ ans & & $0,9419^{* *}$ \\
\hline$\%$ femmes $41-50$ ans & & 0,0339 \\
\hline$\%$ hommes 41-50 ans $=$ ref. & & - \\
\hline$\%$ femmes 51-60 ans & & $-0,1185$ \\
\hline$\%$ hommes 51-60 ans & & 0,3850 \\
\hline$\%$ femmes $61-70$ ans & & $0,7537^{\star *}$ \\
\hline$\%$ hommes 61-70 ans & & 0,3720 \\
\hline$\%$ femmes $71-80$ ans & & 0,4213 \\
\hline$\%$ hommes 71-80 ans & & $-0,0914$ \\
\hline$\%$ femmes $81-90$ ans & & $-0,6642^{* *}$ \\
\hline$\%$ hommes 81-90 ans & & $-0,2187$ \\
\hline \% femmes 91 ans + & & $-0,0420$ \\
\hline$\%$ hommes 91 ans + & & $-0,7965$ \\
\hline \% séjours de sévérité $1=$ ref. & & - \\
\hline \% séjours de sévérité 2 & & $0,8239^{* * *}$ \\
\hline \% séjours de sévérité 3 & & $1,6051^{* * *}$ \\
\hline Admission par transfert $=$ ref. & & - \\
\hline Admission en provenance du domicile & & $-0,1240^{* *}$ \\
\hline Sortie vers un autre service $=$ ref. & & - \\
\hline Sortie vers le domicile & & $-0,0604$ \\
\hline Sortie vers un autre hôpital & & $-0,0667$ \\
\hline Sortie par décès & & $-1,0268^{* * *}$ \\
\hline
\end{tabular}


Tableau 3 (suite)

\begin{tabular}{|c|c|}
\hline & Modèle I Modèle II \\
\hline \% séjours en CMD 1 & $-0,1443$ \\
\hline \% séjours en CMD 2 & $-0,1497$ \\
\hline \% séjours en CMD 3 & $-0,5791^{* *}$ \\
\hline \% séjours en $C M D 4$ & $0,7489^{\star * *}$ \\
\hline \% séjours en CMD 5 & $0,8068^{* * *}$ \\
\hline \% séjours en CMD 6 & $1,6901^{* * *}$ \\
\hline \% séjours en $C M D 8$ & $0,4857^{* * *}$ \\
\hline \% séjours en CMD 14 & $2,0726^{* * *}$ \\
\hline \% séjours en CMD 23 & $0,3671^{\star *}$ \\
\hline$\%$ séjours en hosp. partielle (moins de $24 h$ ) & $0,7965^{\star * *}$ \\
\hline \% séjours chirurgicaux & $0,9670^{* * *}$ \\
\hline Indice de spécialisation & $0,1909^{* * *}$ \\
\hline Intensité de spécialisation & $-0,6586^{* * *}$ \\
\hline $\mathrm{R}^{2}$ & 0,99 \\
\hline
\end{tabular}

1604 hôpitaux 1998-2003, 7731 observations.

MCO appliqués à des spécifications comprenant des effets fixes hôpitaux et des effets fixes temporels (années).

*: significatif $(10 \%), * *$ : significatif $(5 \%)$, ***: significatif $(1 \%)$.

CMD 1: Affections du système nerveux CMD 2: Affections de l'oeil CMD 3: Affections des oreilles, du nez, de la gorge, de la cavité buccale et des dents, CMD 4: affections de l'appareil respiratoire, CMD 5: Affections de l'appareil circulatoire CMD 6: affections du système digestif, CMD 8: Affections de l'appareil musculosquelettique et du tissu conjonctif CMD 14: Grossesse, accouchement et post partum CMD 23: Facteurs influant l'état de santé et autres motifs de recours aux services de santé. Les séjours en hospitalisation partielle (moins de 24H) correspondent souvent à de la chirurgie réalisée en ambulatoire.

Indice de spécialisation $=1$ s'il existe plus de 33\% des séjours au sein du même hôpital dans une CMD donnée, l'intensité est égale à la valeur de cette proportion, si l'indice de spécialisation $=1$, à 0 sinon. 
On obtient un coefficient négatif pour le nombre de lits. En se fondant sur l'expression (3) de l'encadré $C$, une interprétation hâtive conclurait à l'existence de rendements décroissants. Mais une telle conclusion n'est pas légitime, car la spécification comprend un effet fixe, c'est-à-dire une constante spécifique à chaque hôpital qui peut être en rapport avec sa taille. Le résultat ici obtenu signifie seulement que localement, à partir du niveau correspondant à sa constante spécifique, une diminution du nombre de lits conduit à une augmentation de la productivité de l'hôpital'. II ne dit rien sur la taille optimale des établissements, c'est-à-dire sur la productivité comparée, toutes choses égales par ailleurs, d'hôpitaux de différentes tailles ${ }^{8}$.

Le modèle (III) permet d'étudier l'impact des caractéristiques des patients et de l'activité de I'hôpital ${ }^{9}$. On observe que la proportion de patients âgés de plus de 80 ans joue négativement sur la productivité, de façon significative pour les femmes de la tranche 81-90 ans. En revanche, la proportion de femmes de moins de 70 ans, et celle d'hommes jeunes (19-40 ans) jouent positivement. La proportion de patients de degré de sévérité 2 ou 3 a un impact positif sur la productivité. Il faut éviter un contresens sur le contenu de cette variable : par rapport à la catégorie de référence (sévérité 1), les degrés de sévérité 2 ou 3 n'indiquent pas l'existence de comorbidités qui impliqueraient un risque de complication dans la prise en charge du patient. Ils signalent la mise en œuvre d'une procédure chirurgicale intensive ou très intensive. Enfin, on observe que la proportion de patients morts durant leur séjour influence fortement aussi, mais négativement, la productivité de l'hôpital.

Ces résultats montrent comment fonctionne la mesure de la production de l'hôpital à l'aide de la valorisation en points ISA des séjours classés dans les différents GHM. Est favorable à la productivité tout séjour (i) demandant un acte technique assez intensif ; (ii) " simple » au sens premier du terme, c'est-à-dire qui ne correspond qu'à une seule pathologie bien repérée par la nomenclature des GHM. En effet, un acte technique intensif offre une bonne valorisation en nombre de points ISA et un séjour simple est moins long et utilise moins de ressources (pour le même "rendement " en points ISA) qu'un séjour avec des pathologies associées. C'est pourquoi les séjours de personnes très âgées sont défavorables à la productivité : elles ont souvent plusieurs maladies, de nombreux facteurs de complication, et leur fragilité limite l'application de techniques chirurgicales lourdes. Pour la même raison, les degrés de sévérité 2 ou 3 sont très favorables à la productivité, puisqu'ils sont liés au caractère intensif des procédures. Enfin, un patient qui décède à l'hôpital est le cas le plus défavorable: il s'agit souvent de personnes très âgées, affectées de nombreuses pathologies et présentant de gros risques de complications. Beaucoup de ressources sont mobilisées pour un classement dans un seul GHM.

\footnotetext{
${ }^{7}$ Ou, à l'inverse, qu'une augmentation du nombre de lits entraîne une diminution de la productivité de I’hôpital. Mais le scénario de la diminution du nombre de lits est plus conforme aux ajustements observés actuellement.

${ }^{8}$ Pour examiner ce point (rapidement car ce n'est pas l'objet de l'étude), nous avons procédé à l'estimation d'un modèle sans effet fixe avec une forme polynomiale pour affiner l'estimation de l'impact du nombre de lits sur la productivité. Avec un polynôme de degré 3, on obtient par exemple, pour l'échantillon sans les $\mathrm{CHU}$, un minimum local de productivité pour une taille de 8 lits, puis un maximum pour une taille de 253 lits.

${ }^{9}$ Nous ne commentons pas les estimations du modèle (II), qui n'intègre que les caractéristiques des patients et donne pour l'impact de ces variables des résultats très proches de ceux du modèle (III).
} 
Les estimations figurant dans la partie basse du tableau 3 montrent l'influence de la composition de l'activité de l'hôpital sur sa productivité. La proportion de séjours chirurgicaux joue très fortement sur la productivité, ainsi que les séjours en hospitalisation partielle (moins de $24 \mathrm{H}$ ). Les mêmes mécanismes sont à l'œuvre : le chirurgical est bien coté en points ISA et les hospitalisations partielles utilisent moins de ressources. Développer son activité dans certaines directions a aussi un impact sur la productivité : la proportion de séjours classés dans la catégorie majeure de diagnostic relative aux accouchements (CMD14) a un fort impact positif, tout comme la proportion de séjours en cardiaque (CMD5) ou ceux consacrés aux affections du tube digestif (CMD6), aux affections de l'appareil respiratoire (CMD4) ou à l'orthopédie (CMD8).

\section{Efficacité productive des hôpitaux publics, privés et PSPH}

Les estimations permettent d'évaluer pour chaque hôpital la distance $u_{h}$ à la frontière de production efficace définie par la fonction de production. On en déduit un taux d'efficacité productive effi $i_{h}$ défini par : effi $i_{h}=\exp \left\{-u_{h}\right\}$. Ce terme s'interprète de la façon suivante : une valeur de 82,4 (médiane des effi $i_{h}$ obtenue pour les grands hôpitaux publics dans le modèle (I)) signifie que l'hôpital produit seulement $82,4 \%$ de ce qu'il pourrait produire, compte tenu des facteurs de production mobilisés. Nous obtenons une valeur de effi $i_{h}$ pour chacun des 1604 hôpitaux. Les distributions et les médianes des effi $i_{h}$ sont présentées respectivement dans les graphiques 4 et 5 et dans le tableau $4 \mathrm{~b}$ pour les différents modèles considérés, en distinguant les statuts et les tailles des hôpitaux. 
Tableau 4a : Estimation, seconde étape

Estimation de l'équation $\hat{\eta}_{h}=\gamma+\delta . c h u_{h}+v_{h}-u_{h}$

\begin{tabular}{|c|c|c|c|}
\hline & Modèle I & Modèle II & Modèle III \\
\hline \hline $\begin{array}{c}\text { Paramètre d'asymétrie } \\
\lambda=\frac{\sigma_{u}}{\sigma_{v}}\end{array}$ & 3.471 & 2.763 & 1.222 \\
\hline \hline $\begin{array}{c}\text { Risque de première espèce } \\
\text { pour le test de rapport de } \\
\text { vraisemblance de } \mathrm{H}_{0}: \sigma_{u}=0\end{array}$ & 0.000 & 0.000 & 0.000 \\
\hline \hline Coefficient de chu & $0.649^{* * *}$ & $0.694^{* * *}$ & $1.027^{* * *}$ \\
\hline \hline
\end{tabular}

Tableau 4b

Médianes des taux d'efficacité productive estimés $e f f i_{h}$

\begin{tabular}{|c|c|c|c|c|}
\hline Taille & Statut & Modèle I & Modèle II & Modèle III \\
\hline \hline \multirow{3}{*}{ Petit } & Public & 17.2 & 30.2 & 48.2 \\
& PSPH & 43.6 & 50.1 & 64.4 \\
& Privé & 57.9 & 57.0 & 62.9 \\
\hline \hline \multirow{3}{*}{ Moyen } & Public & 64.2 & 74.9 & 78.6 \\
& PSPH & 79.4 & 75.7 & 78.6 \\
& Privé & 80.8 & 80.5 & 76.3 \\
\hline \hline \multirow{3}{*}{ Grand } & Public & 82.4 & 85.9 & 84.5 \\
& PSPH & 87.6 & 85.5 & 83.8 \\
& Privé & 88.7 & 87.4 & 81.7 \\
\hline
\end{tabular}

1604 hôpitaux, 7731 observations dans la dimension hôpital-année ; période 1998-2003.

Le taux d'efficacité est défini pour chaque hôpital $h$ par $\operatorname{eff} i_{h}=\exp \left(-u_{h}\right)=Q_{h} / Q^{\max }{ }_{h}$

Lecture : effi=82.4 est la médiane des effi $i_{h}$ estimés pour les grands hôpitaux publics. Cette valeur signifie que, en valeur médiane, les grands hôpitaux publics produisent seulement $82.4 \%$ de ce qu'ils pourraient produire, compte tenu des facteurs utilisés. 
Graphique 4

Distribution des taux d'efficacité effi $i_{h}$ estimés à partir du modèle (I)

Taux d'efficacité par taille

Small hospitals

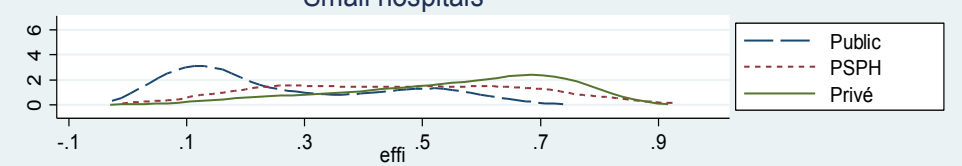

Medium hospitals

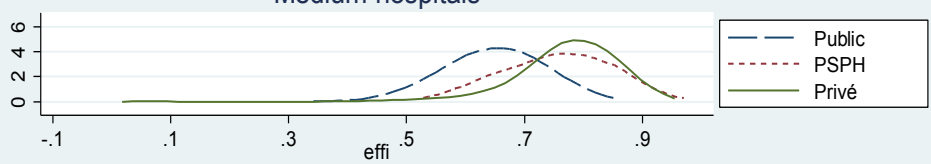

Big hospitals

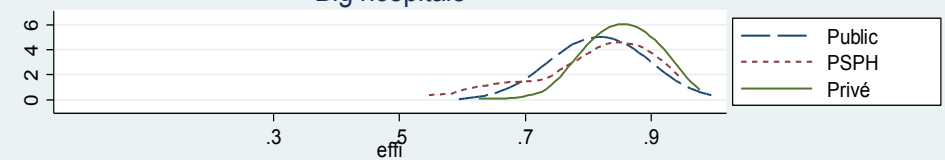

Source: SAE_PMSI 1998-2003

\section{Graphique 5}

Distribution des taux d'efficacité effi ${ }_{h}$ estimés à partir du modèle (III)

\section{Taux d'efficacité par taille}

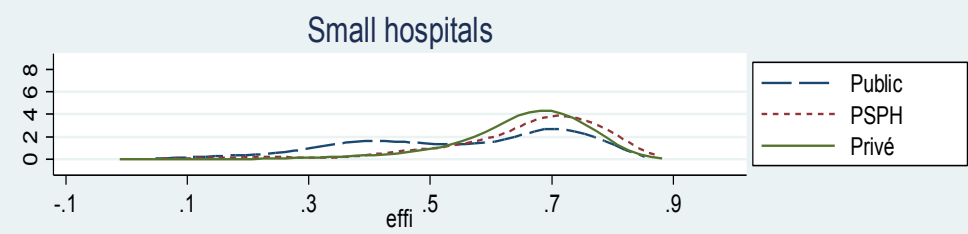

Medium hospitals

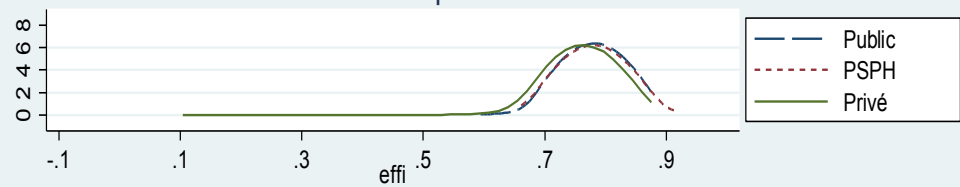

Big hospitals

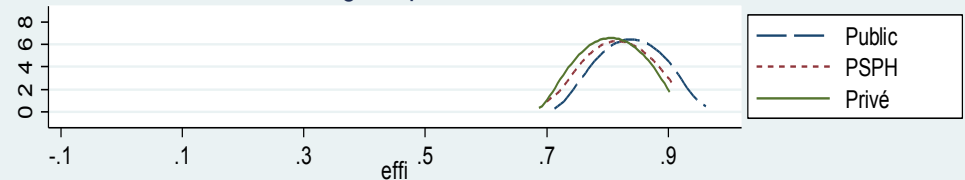

Source: SAE_PMSI 1998-2003 
Les taux d'efficacité productive estimés à partir du modèle (I) font apparaître les hôpitaux publics comme moins efficaces que les hôpitaux privés, quelle que soit leur taille (tableau 4b). L'écart est très marqué pour les petits hôpitaux : les petits hôpitaux publics semblent extraordinairement peu efficaces, avec un taux médian de 17,2 \% ! On obtient des valeurs plus raisonnables pour les taux d'efficacité des autres hôpitaux, avec aussi un écart plus restreint entre les hôpitaux publics et privés de taille moyenne (64,2\% versus $80,8 \%$ ). L'écart d'efficacité se réduit encore pour les grands hôpitaux, mais avec toujours une supériorité du secteur privé $(82,4$ \% pour les grands hôpitaux publics versus 88,7 \% pour les grands hôpitaux privés). Les hôpitaux PSPH présentent des taux d'efficacité de valeur intermédiaire, mais plus proches des taux d'efficacité des cliniques privées que de ceux du public.

Comme nous l'avons déjà expliqué, notre stratégie empirique consiste à examiner comment le diagnostic sur l'efficacité productive varie lorsque l'on incorpore des variables explicatives décrivant les caractéristiques des patients et de la production des hôpitaux. De façon générale, ajouter des variables explicatives à la spécification influence l'évaluation de l'efficacité car elle fait varier la localisation de la frontière. II n'y a pas de raison mécanique permettant de supposer qu'intégrer les caractéristiques des patients et de l'activité des hôpitaux devrait jouer en faveur d'une amélioration des performances mesurées pour le secteur public. On sait toutefois que les hôpitaux privés ont toute latitude pour se positionner sur certains types d'activité et peuvent sélectionner leurs patients. II est vraisemblable que ces stratégies visent à gagner en efficacité. Si ce raisonnement est exact, intégrer ces éléments dans l'évaluation de la frontière de production devrait améliorer la situation relative des hôpitaux publics.

Effectivement, la situation relative des hôpitaux publics s'améliore lorsque l'on intègre les caractéristiques des patients (modèle (II)), et plus encore lorsque l'on ajoute les caractéristiques de l'activité des hôpitaux. Avec le modèle (III), les hôpitaux les plus efficaces sont les hôpitaux publics, au moins en ce qui concerne les établissements de moyenne ou de grande taille ${ }^{10}$. Les graphiques 4 et 5 , qui présentent les distributions des taux d'efficacité estimés avec les modèles (I) et (III) conduisent à la même conclusion: l'efficacité productive des hôpitaux publics est supérieure à celle des cliniques privées lorsque l'on tient compte de la composition des patients et de l'activité de l'hôpital dans la définition de la frontière de production.

\section{Les composantes des différences de productivité des hôpitaux publics et privés}

L'estimation de la fonction de production permet de quantifier les composantes des différences de productivité entre les hôpitaux selon leur statut. Avec trois statuts (publics, privés et PSPH) et trois tailles d'hôpitaux, neuf combinaisons peuvent être examinées pour les contrastes. Les résultats sont publiés dans le tableau 5 pour l'estimation du modèle (III). Les décompositions déduites de l'estimation du modèle de base (I) sont données dans le tableau 6 en annexe: elles conduisent à des résultats identiques pour l'impact des facteurs de production.

\footnotetext{
${ }^{10}$ Les petits hôpitaux publics semblent irrécupérables, même si leur taux d'efficacité s'améliore beaucoup entre les tréfonds du $17,2 \%$ obtenus avec le modèle (I) et les $48,2 \%$ obtenus avec le modèle (III).
} 
Tableau 5 : Décomposition des différences de productivité (à partir du modèle III)

\begin{tabular}{|c|c|c|c|c|c|c|c|c|c|}
\hline & $\begin{array}{c}\text { Petit } \\
\text { Public - Privé } \\
\text { (a) }\end{array}$ & $\begin{array}{c}\text { Moyen } \\
\text { Public - Privé } \\
\text { (b) }\end{array}$ & $\begin{array}{c}\text { Grand } \\
\text { Public - Privé } \\
\text { (c) }\end{array}$ & $\begin{array}{c}\text { Petit } \\
\text { Public - PSPH } \\
\text { (d) }\end{array}$ & $\begin{array}{c}\text { Moyen } \\
\text { Public-PSPH } \\
\text { (e) }\end{array}$ & $\begin{array}{c}\text { Grand } \\
\text { Public - PSPH } \\
\text { (f) }\end{array}$ & $\begin{array}{c}\text { Petit } \\
\text { PSPH-Privé } \\
(\mathrm{g})\end{array}$ & $\begin{array}{c}\text { Moyen } \\
\text { PSPH-Privé } \\
\text { (h) }\end{array}$ & $\begin{array}{c}\text { Grand } \\
\text { PSPH-Privé } \\
\text { (i) }\end{array}$ \\
\hline $\begin{array}{c}\text { Différence } \\
\text { moyenne de } \\
\text { productivité } \\
\text { (à expliquer) (1) }\end{array}$ & -54.5 & -33.6 & -33.7 & - 37.2 & -26.2 & -12.7 & -17.3 & - 7.4 & -21.0 \\
\hline $\begin{array}{l}\text { Due à : } \\
\text { Lits } \\
\text { Infirmières } \\
\text { Aide-soignantes } \\
\text { Pers. administratif } \\
\text { Pers. Medico-tech. } \\
\end{array}$ & $\begin{array}{c}+23.9 \\
9.3 \\
9.9 \\
10.6 \\
-15.1 \\
\end{array}$ & $\begin{array}{c}-12.4 \\
5.5 \\
4.0 \\
5.1 \\
-8.4 \\
\end{array}$ & $\begin{array}{l}-38.1 \\
+5.5 \\
+2.8 \\
+5.4 \\
-9.1 \\
\end{array}$ & $\begin{array}{c}+24.4 \\
4.1 \\
7.5 \\
3.4 \\
-7.7 \\
\end{array}$ & $\begin{array}{l}+0.5 \\
2.2 \\
3.3 \\
-1.6 \\
-2.0 \\
\end{array}$ & $\begin{array}{c}-16.3 \\
2,1 \\
2,1 \\
-2,0 \\
-1.8 \\
\end{array}$ & $\begin{array}{c}-0.5 \\
5.2 \\
2.4 \\
7.2 \\
-7.4 \\
\end{array}$ & $\begin{array}{c}-12.9 \\
3.3 \\
0.8 \\
6.7 \\
-6.4 \\
\end{array}$ & $\begin{array}{c}-21.8 \\
3.4 \\
0.7 \\
7.4 \\
-7.3 \\
\end{array}$ \\
\hline $\begin{array}{l}\text { Diff. totale due } \\
\text { aux facteurs de } \\
\text { production (2) }\end{array}$ & +38.6 & -6.2 & -33.6 & +31.6 & +2.3 & -16.1 & +6.9 & -8.5 & -17.5 \\
\hline $\begin{array}{l}\text { Diff. totale due } \\
\text { aux caract. des } \\
\text { patients (3) }\end{array}$ & -25.8 & -14.1 & -11.1 & -19.9 & - 19.1 & -13.3 & -6.0 & +5.0 & +2.2 \\
\hline $\begin{array}{l}\text { Diff. totale due } \\
\text { aux caract. de la } \\
\text { production (4) } \\
\text { (dont \% séjours } \\
\text { chirurgicaux) } \\
\end{array}$ & $\begin{array}{c}-45.7 \\
(-38.0)\end{array}$ & $\begin{array}{c}-27.5 \\
(-29.0)\end{array}$ & $\begin{array}{l}-29.0 \\
(-25.7)\end{array}$ & $\begin{array}{c}-20.5 \\
(-12.6)\end{array}$ & $\begin{array}{l}-9.4 \\
(-9.0)\end{array}$ & $\begin{array}{l}-8.9 \\
(-6.5)\end{array}$ & $\begin{array}{c}-25.2 \\
(-25.4)\end{array}$ & $\begin{array}{c}-18.1 \\
(-20.0)\end{array}$ & $\begin{array}{c}-20.1 \\
(-19.1)\end{array}$ \\
\hline CHU (5) & +4.4 & +2.6 & 23.7 & +4.4 & +2.6 & 23.7 & - & 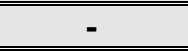 & - \\
\hline $\begin{array}{c}\text { Hétérogénéité } \\
\text { non observée }(6)\end{array}$ & -4.7 & +8.2 & 14.9 & -9.3 & -2.2 & +3.9 & +4.6 & +10.4 & +10.9 \\
\hline Inefficacité (7) & -34.1 & +2.9 & 0.7 & -32.9 & -1.9 & -1.0 & -1.2 & +4.8 & +1.7 \\
\hline Résidu* (8) & 13.0 & 0.6 & 0.7 & 9.4 & 1.5 & -1.1 & 3.6 & -0.9 & 1.9 \\
\hline
\end{tabular}

1604 hôpitaux, 7731 observations dans la dimension hôpital-année ; période 1998-2003. On a : (g) = (a)-(d) ; (h) = (b)- (e); (i) = (c) - (f).

Un résidu apparaît car la procédure en deux étapes est non linéaire 
Examinons tout d'abord, pour les grand hôpitaux, les contrastes entre les hôpitaux publics et privés à but lucratif (tableau 5, colonne (c)). Les hôpitaux publics sont moins productifs que les hôpitaux privés, avec un écart important de 33,7 \%. L'impact des différents facteurs de production sur cet écart est détaillé dans le tableau 5 , puis synthétisé (ligne (2)). Le principal effet négatif vient du nombre de lits (- 38, $1 \%)$, trop important dans le secteur public et du personnel médico-technique (- 9,1\%), vraisemblablement en surnombre. Au total, les différences relatives aux facteurs de production expliquent un écart de $-33,6 \%$ dans la productivité public-privé. Les caractéristiques de la patientèle jouent aussi au détriment de la productivité des hôpitaux publics avec un impact de - 11,1\%. Les caractéristiques de la production nuisent aussi fortement à la productivité des hôpitaux publics : elles expliquent un écart négatif de $29 \%$ par rapport au privé, dont $25,7 \%$ sont dus à la proportion de séjours chirurgicaux. La spécialisation du secteur privé dans les séjours chirurgicaux explique une grande partie de son avantage comparatif en matière de productivité. A contrario, l'impact des centres hospitalo-universitaires, hôpitaux à haute technologie présents dans le seul secteur public, et celui de l'hétérogénéité non observée jouent en faveur de la productivité dans le public. L'influence de l'hétérogénéité non observée reflète celle de variables omises comme les médecins, voire la présence d'économie d'échelle ou d'économie de gamme. Enfin, comme nous l'avons déjà vu, les différences d'efficacité productive jouent en faveur du secteur public (tableau 5, colonne (c), ligne (7)). Cet effet est faible toutefois $(+0,7 \%)$.

Les hôpitaux privés à but non lucratif sont peu développés en France, alors que ce statut domine dans le secteur hospitalier américain (Etats-Unis). Certains cherchent à promouvoir cette forme juridique pour les hôpitaux français, arguant qu'elle permet d'allier la souplesse de gestion du privé à une fonction objectif compatible avec un cahier des charges conforme à l'intérêt public (Silber, 2005). Quelle est la performance des grands hôpitaux PSPH, par rapport à celle des grands hôpitaux privés? Ils sont moins productifs, avec un écart de $21,0 \%$ (tableau 5 , colonne (i)). Pour être important, cet écart est toutefois est plus modéré que celui observé entre les grands hôpitaux publics et privés. On retrouve les mêmes facteurs handicapant la productivité des grands hôpitaux PSPH que ceux observés pour les grands hôpitaux publics : nombre de lits (perte de productivité de $21,8 \%)$, personnel médico-technique (- 7,3\%), composition de la production (- 20,1\%, dont 19,1\% dus à la proportion de séjours chirurgicaux). On retrouve aussi l'impact positif de l'hétérogénéité non observée et une influence positive, mais faible, de l'efficacité productive $(+1,7 \%)$. Le seul résultat nouveau - mais remarquable - réside dans l'impact de la composition de la patientèle, qui joue ici en faveur de la productivité des hôpitaux PSPH, contrairement à ce que l'on observait pour les hôpitaux publics. Tout se passe comme si les hôpitaux PSPH étaient similaires aux hôpitaux publics, à ceci près qu'ils bénéficient d'une sélection de patients qui leur est avantageuse, leur conférant même un gain par rapport au privé à but lucratif (+ 2,2\%). Comme les hôpitaux PSPH n'ont pas le droit de sélectionner leurs patients, les mécanismes d'une telle sélection restent à explorer, qu'il s'agisse de stratégies de ciblage de l'offre de soins, d'un lien privilégié de l'établissement avec une mutuelle ou des comportements d'adressage des médecins.

Etudier les contrastes entre les hôpitaux de taille moyenne conduit aux mêmes conclusions. Les hôpitaux PSPH et privés sont plus nombreux dans cette catégorie (234 hôpitaux privés et 40 PSPH, tableau 1) que dans la catégorie des grands hôpitaux: retrouver les mêmes constats permet d'asseoir la robustesse des comparaisons réalisées. Seule différence notable, les hôpitaux publics et PSPH apparaissent dotés d'un nombre de lits moins défavorable, entraînant un écart de productivité vis-à-vis du privé moins important, d'environ 12 points (colonnes (b) et (h), ligne " lits »). L'écart de 
productivité des hôpitaux PSPH avec les hôpitaux privés est plus faible $(-7,4 \%$, tableau 5 , colonne (h)) que celui observé entre les grands hôpitaux de ces mêmes catégories (- 21,0 \%). Ce rapprochement des performances s'explique par le nombre de lits et par une composition de la patientèle encore plus favorable que celle observée pour les établissements de grande taille, conférant aux hôpitaux PSPH un avantage relatif de $+5,0 \%$ en matière de productivité.

La performance des petits hôpitaux publics est très mauvaise par rapport à celle des petites cliniques privées: on observe un écart de productivité de $-54,5 \%$ (tableau 5 , colonne (a)). Cet écart s'explique à la fois par la composition de leur patientèle (- 25,8 \%), les caractéristiques de leur activité $(-45,7 \%)$ et une grande inefficacité ( - 34,1 \%). Les petits hôpitaux PSPH réalisent une moins mauvaise performance, avec un écart de productivité de $-17,3 \%$ avec les cliniques privées. Cette fois-ci la composition de leur patientèle ne leur est pas favorable, avec un impact de $-6,0 \%$ sur leur productivité (tableau 5 , colonne (g)), contrairement à ce que l'on observait pour les hôpitaux PSPH de grande ou moyenne taille.

\section{Conclusions : les enjeux de la convergence tarifaire}

L'introduction de la T2A vise à instaurer un mécanisme de concurrence par comparaison pour obtenir des gains d'efficacité dans la production de soins hospitaliers. La convergence tarifaire appliquerait ce mécanisme de concurrence à un périmètre englobant les secteurs public et privé de I'hospitalisation. Elle aurait pour objectif de réduire les différences de coûts de traitement observées entre ces deux secteurs. Ces différences de coûts peuvent être dues à des écarts de productivité ou, à niveau de productivité donné, à des différences dans les coûts des facteurs. Elles peuvent aussi résulter d'une inefficacité allocative si les hôpitaux n'ajustent pas les proportions de facteurs qu'ils utilisent en fonction de leurs coûts relatifs.

D'où peuvent venir les écarts de productivité ? Si les hôpitaux procèdent à une optimisation de leur organisation, ils peuvent apparaître à cause de coûts des facteurs différents entre les secteurs. Mais ils peuvent aussi découler d'une inefficacité productive si la production effectivement réalisée est inférieure à la frontière définie par la fonction de production, c'est-à-dire à la production potentielle, compte tenu des facteurs utilisés.

Se focaliser sur la productivité revient à n'examiner qu'une partie des causes potentielles des écarts de coûts public-privé. Mais cette approche offre deux avantages : elle permet d'utiliser un indicateur fiable et comparable pour les secteurs public et privé ; elle permet de réaliser les estimations sur des données représentant quasi exhaustivement les hôpitaux français. De toute façon, il serait difficile d'obtenir des résultats crédibles en travaillant sur les coûts : seul un échantillon restreint de quelques dizaines d'hôpitaux dispose d'information sur les coûts des séjours et il n'existe pas encore de mesure des coûts établie de manière comparable pour les hôpitaux publics et privés ${ }^{11}$.

Nous montrons que le diagnostic sur l'efficacité productive des hôpitaux publics dépend de la définition de la frontière de production : lorsque l'on utilise une fonction de production classique mettant en relation les inputs et l'output, les scores d'efficacité des hôpitaux publics sont inférieurs à

\footnotetext{
${ }^{11}$ Malgré l'importance des efforts fournis dans ce sens (ATIH, 2010).
} 
ceux des hôpitaux PSPH, eux-mêmes inférieurs à ceux des cliniques privées. Cette hiérarchie dans la performance est observée quelle que soit la taille des établissements. Mais l'ordre des performances relatives s'inverse lorsque l'on intègre à la spécification estimée les caractéristiques de la patientèle et des indicateurs décrivant la composition des séjours : à l'exception des petits établissements, les hôpitaux publics apparaissent alors plus efficaces que les cliniques privées. C'est aussi le cas des hôpitaux PSPH, qui apparaissent plus efficaces que les cliniques privées quelle que soit leur taille lorsque l'on tient compte des caractéristiques des patients et des séjours. Ce résultat sur la hiérarchie des taux d'efficacité est obtenu de façon robuste pour de nombreuses variantes concernant la spécification de la fonction de production, l'inclusion ou non des $\mathrm{CHU}$ dans les données, l'introduction ou non des médecins dans les variables explicatives, etc.

Ces résultats doivent être interprétés à la lumière des différences de cahiers des charges encadrant les activités des hôpitaux publics et privés. II est frappant de constater que la prise en compte d'indicateurs concernant la structure de l'activité et la composition de la patientèle modifie radicalement le diagnostic sur l'efficacité productive des hôpitaux publics. En effet, sur la période considérée, structure de l'activité et composition de la patientèle sont déterminées de façon exogène aux établissements publics dans le cadre de la réponse aux besoins que constitue le service public hospitalier.

Nos estimations permettent aussi d'évaluer les composantes des différences de productivité entre les hôpitaux de différents statuts. La plus faible productivité des hôpitaux publics s'explique principalement par le nombre de lits, des personnels médico-techniques en sureffectifs, la composition de leur patientèle et celle de leurs séjours (caractérisée entre autres par une faible proportion de séjours chirurgicaux). Elle ne s'explique pas, comme nous l'avons vu, par une moindre efficacité des hôpitaux publics ${ }^{12}$.

La concurrence avec le secteur privé qui serait introduite par la convergence tarifaire pourrait inciter les hôpitaux publics à ajuster leur nombre de lits et les effectifs de certaines catégories de personnel. Encore faudrait-il que ce soit possible : des marges de manœuvre doivent être données aux hôpitaux publics pour disposer d'un minimum de flexibilité dans la gestion de leur personnel et des facteurs de production.

II faudrait aussi que ce soit nécessaire. En effet, c'est l'égalisation des rapports des productivités marginales des facteurs de productions et de leurs coûts relatifs qui détermine la minimisation du coût. Une évaluation récente réalisée pour l'année 2007 a montré que les salaires bruts des personnels hospitaliers étaient plus élevés dans les cliniques privées (et plus encore dans les hôpitaux privés à but non lucratif) que dans le secteur public (Aude et Raynaud, 2009). Dans cette hypothèse, une moindre productivité des personnels des hôpitaux publics est compatible avec une minimisation du coût ${ }^{13}$.

Nous avons vu que le diagnostic sur l'efficacité productive des hôpitaux est influencé par la composition de leur patientèle et de leur activité. Leur productivité est aussi influencée par ces mêmes éléments. Ce résultat est préoccupant. II implique en effet que la convergence tarifaire

\footnotetext{
${ }^{12} \mathrm{~A}$ l'exception des petits établissements (ayant moins de 5000 séjours par an).

${ }^{13}$ Il faudrait évidemment examiner cette idée plus attentivement en estimant les demandes de facteurs émanant des hôpitaux.
} 
devrait inciter les hôpitaux publics à manipuler la composition de leur patientèle et à restructurer leur offre de soins : en se basant sur les estimations, cela reviendrait par exemple à accueillir moins de femmes de plus de 80 ans, et à réaliser plus de séjours chirurgicaux. Dans le système de la T2A, les poids $p_{j t}$ que nous utilisons pour construire notre mesure de la productivité servent à calculer les tarifs. Ils fonctionnent donc comme un vecteur de prix qui orientent la structuration de l'offre de la part des producteurs de soins que sont les hôpitaux. Or, ces poids $p_{j t}$ sont déterminés par l'administration sur la base des coûts relatifs de traitement des séjours dans les différents GHM. Ils ne sont pas déterminés, du côté de la demande, par la valeur sociale, basée sur les préférences collectives, attribuée aux soins délivrés pour un séjour dans un GHM donné.

Dans ce cadre, la T2A, si elle permet d'inciter à l'efficacité productive, pourrait conduire à une réorientation de l'offre de soins dans un sens non conforme aux besoins. Dans cette hypothèse, elle pourrait nuire à l'efficacité allocative.

\section{Bibliographie}

Aballea, P., Bras, P.-L. et S. Seydoux, (2006). Mission d'appui sur la convergence tarifaire public privé, rapport IGAS n²006 009 http://lesrapports.ladocumentationfrancaise.fr/BRP/064000080/0000.pdf

Aigner, D.J., Lovell, C.A.K. and Schmidt P. (1977) Formulation and Estimation of Stochastic Frontier Production Function Models, Journal of Econometrics, Vol.6, 21-37.

Arnault, S., F. Evain, A. Fizzala and I. Leroux, 2009. L'activité des établissements de santé en 2007 en hospitalisation complète et partielle, Etudes et Résultats $n^{\circ} 691$, mai.

ATIH (2010) Guide de l'Etude Nationale de Coûts à méthodologie Commune MCO, http://www.atih.sante.fr/?id=0000F00001FF

Aude J. et D. Raynaud (2009), « Les écarts de salaires bruts entre hôpitaux publics et cliniques privées en 2007 ", Économie publique, 24-25 | 2009/1-2

Battese, G. and Coelli T., 1992. Frontier production functions, technical efficiency and panel data: with application to paddy farmers in India., Journal of Productivity Analysis ; 3: 153--169.

Battese, G. and Coelli T., 1995. A model for technical inefficiency effects in a stochastic frontier production function for panel data. Empirical Economics ; 20:325--332.

Burgess J. F., and P. W. Wilson (1996), Hospital Ownership and Technical Inefficiency, Management Science, Vol. 42, No. 1, pp. 110-123

Cour des Comptes (2009) La Sécurité sociale, rapport, septembre http://www.ccomptes.fr/fr/CC/documents/RELFSS/Rapport-securite-sociale2009.pdf

DHOS (2009). Rapport 2009 au Parlement sur la convergence tarifaire. http://www.sante.gouv.fr/IMG/pdf/Rapport convergence 2009.pdf

DREES (2011) Les comptes nationaux de la santé, Collection Etudes et statistiques 
Gobillon L. et C. Milcent (2010) Innovative Procedures: the key factor for hospital performance, Working Paper

Hart, O., Shleifer, A., Vishny, R.W., 1997. The proper scope of government: theory and an application to prisons. Quarterly Journal of Economics , 112 (4), 1127--1162.

Herr A., Schmitz H. et Augurzky B. (2010) Profit efficiency and ownership of German hospitals. Health Economics, vol. 17(9), pages 1057-1071

Jondrow J., Lovell C.A.K.,Materov I.S. and Schmidt P. (1982) On the Estimation of Technical Inefficiency in the Stochastic Frontier Production Function Model, Journal of Econometrics, Vol.19, 233-238

Lakdawalla, D. and T. Philipson, 2006. The nonprofit sector and industry performance, Journal of Public Economics 90, 1681-1698

Lien, H.-M., S.-Y. Chou and J.-T. Liu, 2008. Hospital ownership and performance: Evidence from stroke and cardiac treatment in Taiwan, Journal of Health Economics 27, 1208-1223

Milcent C. (2005) : Hospital Ownership, Reimbursement Systems and Mortality Rates", Health Economics, Vol 14 (11), pp.

Newhouse, J.P., 1970. Toward a theory of nonprofit institutions: an economic model of a hospital. American Economic Review, 60 (1), 64--74.

Picone, G.A., Chou, S.-Y., Sloan, F.A., 2002. Are for-profit hospital conversions harmful to patients and to medicare? Rand Journal of Economics, 33 (3), 507--523.

Rosko, M. 2001. Cost efficiency of US hospital: A Stochastic Frontier Approach, Health Economics, Vol 10: pp 539-551

Shleifer, A., 1985. A theory of Yardstick Competition, Rand Journal of Economics, vol 16: pp 319-327

Sloan, F., 2000. Not-for-profit ownership and hospital behavior. In: Culyer, Newhouse (Eds.), Handbook of Health Economics. North Holland.

Sloan, F.A., Picone, G.A., Taylor Jr., D.H., Chou, S.-Y., 2001. Hospital ownership and cost and quality of care: is there a dime's worth of difference? Journal of Health Economics 20 (1), 1--21.

Silber D. (2005) Hôpital : le modèle invisible, note de l'Institut Montaigne, juin http://www.institutmontaigne.org/medias/documents/hopital_le_modele_invisible.pdf 


\section{Encadré A \\ La réforme de la tarification hospitalière en France}

Pourquoi faut-il réguler l'hôpital ? Cet impératif n'est pas lié au statut - public ou privé - des établissements. II résulte de la couverture des soins hospitaliers par une assurance maladie financée par des prélèvements obligatoires. II faut garantir un bon usage des fonds ainsi collectés, autrement dit rechercher l'efficacité de la dépense.

Trois parties sont en présence : les patients, les hôpitaux et la tutelle. Les patients sont aussi des assurés sociaux qui financent le système par leurs contributions. Selon le pays considéré, la tutelle peut s'incarner dans l'État, dans un assureur unique comme la sécurité sociale, ou dans des assurances plurielles en concurrence. La tutelle est censée agir dans l'intérêt des assurés. Elle doit encourager les hôpitaux à répondre aux besoins, sans induire d'activité injustifiée, les inciter à fournir les soins au moindre coût, sans sacrifier la qualité des soins et sans sélectionner les patients. Elle doit encore garantir l'équité dans l'accès aux soins et éviter tout rationnement.

La difficulté vient du fait que la tutelle a des problèmes d'information : elle ne peut observer ni contrôler les efforts fournis par les agents hospitaliers pour réduire les coûts. Elle ne connaît pas bien non plus les contraintes spécifiques auxquelles les hôpitaux peuvent être confrontés. Comment inciter les établissements à l'efficacité productive sans leur demander l'impossible ? Le système de tarification cherche à résoudre ces problèmes.

\section{Paiement prospectif ou rétrospectif}

La littérature économique distingue classiquement paiement prospectif et rétrospectif. Un paiement rétrospectif se borne à rembourser les coûts de traitement constatés ex post, ce qui n'incite pas à un fonctionnement efficace. A l'inverse, un paiement prospectif est fixé sous la forme d'un forfait en rapport avec les besoins du patient tels qu'ils découlent du diagnostic lors de son admission. Un tel paiement incite puissamment à l'efficience car il est indépendant des efforts de réduction des coûts et des décisions de traitement prises lors du séjour hospitalier. La différence entre le forfait et le coût de traitement effectif est à la charge de l'hôpital : c'est un surplus (qu'il peut s'approprier) s'il est efficace, une perte (qu'il subit) dans le cas contraire.

Quatre systèmes de paiement peuvent être considérés. Le paiement à l'acte finance de manière rétrospective les soins au niveau du coût effectivement observé. Le système du budget global consiste à attribuer à l'établissement, selon des critères qui peuvent varier, un budget pour l'ensemble de son activité annuelle. La tarification par pathologie consiste à financer chaque cas traité par un forfait calculé de façon prospective pour chaque pathologie. Le principe de l'achat de soins, enfin, sélectionne par un mécanisme d'enchères le prestataire d'un panier de soins prédéfinis.

En théorie, le budget global et la tarification par pathologie sont de nature prospective. En pratique les modalités de leur application peuvent leur conférer un caractère rétrospectif. Concernant la tarification par pathologie, nous verrons plus loin qu'elle peut facilement se rapprocher d'un paiement à l'acte. Quant au budget global, tout dépend de la rigueur avec laquelle la contrainte budgétaire est imposée : on glisse très rapidement vers un système rétrospectif si les déficits ne menacent aucunement la survie de l'établissement ou l'organisation de sa gouvernance. Pareillement, un important pouvoir de négociation du directeur de l'hôpital auprès de la tutelle pour influencer le budget alloué remet en cause les vertus incitatives d'un budget global. 


\section{Principe de la tarification par pathologie}

L'idée de financer chaque cas traité par un forfait calculé de façon prospective pour chaque pathologie laisse entière la question de la fixation de ces forfaits.

La modélisation retenue suppose en général que le coût de traitement d'un patient atteint d'une pathologie particulière dans l'hôpital $h$ est donné par : $C_{h}=c_{h}-e_{h}$, $c_{h}$ et $e_{h}$ sont des informations privées de l'hôpital : $c_{h}$ est un paramètre technologique qui reflète sa productivité ; $e_{h}$ est l'effort de réduction du coût. L'objectif du régulateur est d'inciter l'hôpital à fournir le niveau d'effort maximal afin de gagner en efficacité. Avec la tarification par pathologie, le régulateur propose un paiement forfaitaire défini indépendamment du coût effectif : dans ce cadre, l'hôpital a intérêt à fournir l'effort optimal afin de réduire ses coûts au maximum.

A ce stade, une partie seulement du problème est résolue. En effet, $c_{h}$ est une information privée de I'hôpital : la définition du forfait par la tutelle peut le conduire à la faillite ou lui attribuer des rentes excessives. Concrètement, cette éventualité peut prendre la forme d'un plaidoyer du directeur de l'hôpital en faveur de tarifs plus généreux, avec des arguments que la tutelle n'a pas les moyens de vérifier. Le problème de la tutelle est ainsi de trouver le niveau de paiement correspondant à la production efficace, autrement dit de définir une formule de tarification permettant d'extraire la totalité de la rente informationnelle, ou de la réduire le plus possible.

Ce problème informationnel peut être résolu en retenant une hypothèse simplificatrice d'homogénéité des hôpitaux : $c_{h}=c, \forall$ h (Shleifer [1985]). Dans ce cas, les disparités de coûts sont exclusivement dues aux différences d'efforts fournis par les établissements pour améliorer leur efficacité : $C_{h}=c-e_{h}$. Un mécanisme de concurrence par comparaison permet alors la révélation du coût de production efficace $c$. Celui-ci consiste à proposer à chaque hôpital un paiement défini sur la base de la moyenne des coûts observés pour les autres établissements. Concrètement, la tutelle propose la règle de paiement suivante à chaque hôpital $h$ au début de l'année $t$ : «Pour chaque séjour dans une pathologie donnée, définie par un groupe homogène de malades (GHM) $g$, vous recevrez un paiement égal au coût moyen par séjour dans ce GHM. La moyenne sera calculée à la fin de l'année sur tous les hôpitaux autres que votre établissement. ". Avec une telle règle de fonctionnement, chaque manager sait qu'il a intérêt à fonctionner efficacement, car il risque sinon d'avoir en fin d'année un coût au dessus du forfait et d'être en déficit. Au final, la moyenne des coûts doit correspondre au coût de fonctionnement efficace.

Cette représentation idéale constitue le fondement théorique de la tarification par pathologie. Elle repose sur des hypothèses peu réalistes : homogénéité des hôpitaux, homogénéité des patients pour une pathologie donnée, qualité des soins fixée. Elle conduit à définir une tarification au forfait très homogène dont les risques sont maintenant connus : sélection ou discrimination des patients, baisse de la qualité des soins délivrés (Newhouse [1996]). Les chercheurs ont tenté d'améliorer le modèle de base en remettant en cause ces hypothèses d'homogénéité. Avec des approches théoriques très différentes, les nouveaux modèles proposent des paiements mixtes qui combinent forfait et coûts observés. Le principe d'un système de paiement mixte fait maintenant l'objet d'un consensus, rejoignant ainsi la pratique de la régulation aux Etats-Unis. Mais le coefficient de partage entre forfait et coût effectif est défini de façon très différente selon le corps d'hypothèses du modèle auquel on se réfère. Le débat sur ces questions reste ouvert : comment identifier le niveau des coûts correspondant à une activité efficace ? Comment intégrer I'hétérogénéité des patients et celle des établissements dans la tarification des séjours hospitaliers? 
La tarification hospitalière en France depuis 1945

Comme dans la plupart des pays de I'OCDE, les hôpitaux français ont tout d'abord été financés par un paiement rétrospectif. Entre 1945 et 1983, celui-ci a pris la forme d'un prix de journée. L'absence de contrainte budgétaire qui a prévalu durant cette période s'est traduite par une forte dérive des dépenses hospitalières. Le système du budget global a été instauré en 1983, avec pour chaque hôpital un budget établi par l'application d'un taux directeur à la dotation de l'exercice précédent. Ce dispositif a contribué à figer les inégalités de dotations initiales indépendamment de l'évolution de l'activité des hôpitaux. Par ailleurs le budget global s'est rapproché d'un paiement rétrospectif car aucun déficit ne menaçait sérieusement la pérennité d'un établissement. Tel qu'il fut appliqué en France, le budget global, a laissé perdurer de nombreuses inefficacités et une allocation des ressources inéquitable.

Conscientes de ces imperfections, les autorités de régulation ont très vite cherché à mettre en place une collecte d'informations sur l'activité de production de soins des établissements. Le lancement en 1982 du Programme de médicalisation des systèmes d'informations (PMSI) répond à cet objectif. Opérationnel à partir de 1994 seulement, le PMSI permet d'évaluer les services rendus par les hôpitaux en nombre de points ISA (indice synthétique d'activité). Dans le PMSI, les séjours sont classés en Groupes homogènes de malades (GHM) à l'aide d'une arborescence sur la base des différents diagnostics et actes pratiqués sur le patient.

Dans le sillage du Plan Juppé, les ordonnances de 1996 ont organisé le vote annuel, dans le cadre de la loi de financement de la Sécurité sociale, de l'Objectif national des dépenses d'Assurance maladie (ONDAM). Cette enveloppe fermée, en principe contraignante, comporte un volet dévolu aux dépenses hospitalières. La Tarification à l'activité (T2A) est introduite à partir de 2004 pour les soins aigus en Médecine, chirurgie et obstétrique. Au départ le budget effectif des hôpitaux publics résultait d'un panachage du budget global et de la tarification par cas. Depuis 2008 la T2A est pleinement appliquée, avec éventuellement un coefficient correcteur pour lisser sur plusieurs années les adaptations nécessaires.

\section{La T2A en pratique}

La T2A s'inspire de la tarification à la pathologie : un paiement forfaitaire est prévu par séjour dans une pathologie donnée, la pathologie étant caractérisée par le GHM dans lequel le séjour est classé. Le mécanisme en place s'inspire du principe de la concurrence par comparaison : tout surcoût non justifié est interprété comme de l'inefficacité et les hôpitaux dont les coûts sont au-dessus de la moyenne sont mis en déficit. Les bilans réalisés font état d'une réduction progressive de la proportion d'hôpitaux en déficit, de $60 \%$ en 2006 à $37 \%$ en 2009.

En pratique, les modalités d'application de la T2A font douter d'obtenir un jour les effets bénéfiques que favoriserait une véritable tarification par pathologie. La classification des GHM étant très dépendante des actes pratiqués, on se rapproche d'un paiement à l'acte. De surcroît, l'ONDAM institue une enveloppe fermée ce qui se traduit concrètement par un mécanisme de point flottant : pour respecter la contrainte budgétaire définie au niveau global, les tarifs unitaires sont ajustés à la baisse en proportion des écarts d'activité non anticipés par l'ONDAM. Ceci crée des incitations à une activité excessive : les hôpitaux ont intérêt à multiplier les actes ou à délivrer des actes plus intensifs que nécessaire. Ce mécanisme délétère désavantage les hôpitaux vertueux qui voudraient rester sobres en matière de choix de traitement car tous subissent la baisse de tarifs engendrée par une augmentation d'activité.

La situation est aggravée par une décision récente d'affinement de la nomenclature utilisée pour le classement des séjours : on est passé en 2009 de la version 10 de la classification, qui comportait 780 GHM, à 2291 GHM dans la version 11. Avec l'introduction de quatre niveaux de sévérité pour la plupart 
des GHM, la France est désormais dotée d'une nomenclature qui figure parmi les plus fines du monde. Le degré de sévérité étant un élément difficilement vérifiable par un éventuel contrôleur, un tel affinement de nomenclature instaure une incitation à surcoder les séjours, qui devrait se traduire par une suractivité apparente non justifiée. Les conséquences financières de ce surcodage sont, certes, désamorcées par le point flottant. Mais en retour, le mécanisme du point flottant décuple l'incitation au surcodage. On s'éloigne fortement d'un système de paiement délivrant des incitations à l'efficacité productive.

En outre, la T2A prévoit de nombreux paiements annexes pour des tâches transversales correspondant à des missions d'intérêt général : enseignement et recherche, Samu, centre antipoison, équipes mobiles (soins palliatifs, gériatrie, etc.), forfaits annuels pour les urgences, ou les greffes. II existe aussi des paiements additionnels pour les dispositifs médicaux implantables et les médicaments onéreux, ainsi que pour les séjours extrêmes dont la durée est atypique. Concentrées dans le secteur public, ces dotations spécifiques sont d'un montant très élevé : en 2007, 63 \% seulement des dépenses au titre de la T2A correspondaient au paiement des séjours proprement dits, le reste étant attribué à ces paiements annexes (Cour des Comptes, 2009). Plus rapide que celui des financements dévolus aux séjours, le développement de ces paiements peut avoir des effets pervers. Venant en déduction de l'ONDAM, ils réduisent l'enveloppe dédiée au paiement des séjours pour tous les hôpitaux, alors que les modalités de fixation de leur niveau ne sont pas clairement établies et qu'aucun mécanisme n'est prévu pour maîtriser leur progression, ce qui contredit la logique poursuivie avec la mise en place de la T2A.

Une autre question importante est celle de la convergence des tarifs des secteurs public et privé, prévue à I'horizon 2018. Nous ne nous étendons pas ici sur cette question : elle est abordée au cœur de notre article, puisqu'il est consacré à la comparaison des performances des hôpitaux publics et privés.

Par rapport au système préexistant du budget global, il n'y a pas de doute que la T2A a permis d'améliorer le financement des hôpitaux. Mais les mécanismes effectivement mis en place s'éloignant beaucoup des principes d'une tarification prospective par pathologie, l'impact de cette réforme sur l'efficacité productive des hôpitaux n'est pas certain. Par ailleurs, des questions importantes restent en suspens : comment prendre en compte l'hétérogénéité des hôpitaux ? Comment, dans le cadre de la T2A, piloter l'offre de soins afin qu'elle réponde aux besoins ? Enfin, la tarification par pathologie ne s'intéresse qu'à l'efficacité productive. Compte tenu des incitations mises en place avec la T2A, il faut impérativement développer des indicateurs pour suivre l'évolution de la qualité des soins dans ce contexte. 


\section{Encadré B}

\section{Les données}

Les données utilisées proviennent de deux bases administratives : les données du PMSI et celles de la SAE.

- Le PMSI, ou Programme de Médicalisation des Systèmes d'Informations, offre une information sur l'activité des hôpitaux pour les soins aigus. L'information est collectée au niveau des séjours hospitaliers pour le champ des séjours en médecine, chirurgie obstétrique (MCO), et ce pour l'ensemble des hôpitaux français. La participation des hôpitaux à ce système d'information est obligatoire. Seuls sont exclus les hôpitaux hors MCO, lesquels correspondent souvent à de petits hôpitaux publics locaux.

Pour chaque séjour, les informations disponibles sont : le GHM dans lequel le séjour a été classé, le diagnostic principal, les éventuels diagnostics secondaires, les actes réalisés, le mode d'entrée (provenant du domicile, d'un autre service, d'un autre hôpital), le mode de sortie (retour au domicile, vers un autre service, vers un autre hôpital, décès), la durée de séjour, le sexe et l'âge du patient.

- La SAE, ou Statistique annuelle des établissements de santé, recueille des informations au niveau de l'hôpital-année sur le nombre de lits, leur taux d'occupation, le nombre d'entrées, les équipements, le nombre de médecins, d'infirmier(e)s, d'aides-soignant(e)s, le personnel administratif et le personnel médico-technique. En principe, le personnel est mesuré en équivalent-temps-plein.

Les deux bases sont regroupées en une seule. Ce faisant, nous avons agrégé au niveau de l'hôpital les informations provenant de la base PMSI, qui sont situées au niveau du séjour. Ont été éliminés les hôpitaux dont le code d'identification n'était pas disponible, ainsi que les hôpitaux n'ayant pas de personnel ou pas de lits (il s'agit généralement de petits hôpitaux prenant en charge exclusivement des séances de chimiothérapie, radiothérapie ou dialyse).

La base finale contient 1604 hôpitaux sur la période 1998-2003. Les 7731 observations se situent au niveau hôpital-année. Ce panel est non cylindré : un hôpital donné n'est pas systématiquement observé pour chaque année sur la période 1998-2003. Cette base est proche de l'exhaustivité pour l'activité de type MCO : pour l'année 2003, elle représente $90 \%$ de l'ensemble des admissions pour MCO en France métropolitaine. Le tableau 1, commenté dans le corps de l'article, donne des éléments descriptifs sur les hôpitaux français ayant une activité de type $M C O$, tels qu'ils apparaissent dans les données.

Le nombre de médecins n'est pas renseigné dans la SAE pour 435 hôpitaux privés ou PSPH : ceci correspond au cas où tous les médecins intervenants de l'hôpital sont des médecins libéraux. L'existence d'intervenants libéraux suggère que le nombre de médecins intervenants dans les cliniques privées ou les hôpitaux PSPH est sous estimé. Enfin le calcul en " équivalent-temps-plein » 
(ETP) du nombre de médecins est relativement héröque en dehors du secteur public. En effet, le pourcentage d'heures travaillées par les médecins intervenant dans les hôpitaux privés ou PSPH n'est pas renseigné, alors que l'on sait que dans la réalité les interventions peuvent être régulières ou extrêmement ponctuelles.

Ces raisons font qu'il est plus raisonnable de traiter le nombre de médecins comme une variable omise dans les spécifications économétriques. Soulignons que l'exercice libéral de la médecine conduisent aux mêmes difficultés sur données étrangères (par exemple américaines, voir Burgess et Wilson, 1996), avec la même stratégie empirique pour en tenir compte. 
Encadré C :

Spécification et estimation économétrique

Spécification

Pour étudier l'efficacité productive des hôpitaux, nous considérons une fonction de production de Cobb-Douglas avec six facteurs de production : le nombre de lits, lit, de médecins, med, le nombre d'infirmièr(e)s, inf, le nombre d'aides-soignant(e)s, as, le personnel administratif $a d m$ et le personnel médico-technique, tech. La production est définie par le nombre de points ISA et notée $Q_{h t}$ pour I'hôpital $h$ observé en $t$. On a :

$$
Q_{h t}=A\left(\operatorname{med}_{h t}\right)^{\alpha_{1}}\left(\text { inf }_{h t}\right)^{\alpha_{2}}\left(a s_{h t}\right)^{\alpha_{3}}\left(a d m_{h t}\right)^{\alpha_{4}}\left(t e c h_{h t}\right)^{\alpha_{5}}\left(l i t_{h t}\right)^{\beta}
$$

En prenant les logarithmes on aboutit à une expression linéaire expliquant la productivité de I'hôpital, définie par le nombre de points ISA par lit, par le logarithme du nombre de lits, noté $l_{h t}$, et par les logarithmes des différents facteurs de production, rapportés au nombre de lits :

$$
\begin{gathered}
q_{h t}-l_{h t}=(\mu-1) l_{h t}+\alpha_{1}\left[\log \operatorname{med}_{h t}-l_{h t}\right]+\alpha_{2}\left[\log i n f_{h t}-l_{h t}\right]+\alpha_{3}\left[\log a s_{h t}-l_{h t}\right] \\
+\alpha_{4}\left[\log a d m_{h t}-l_{h t}\right]+\alpha_{5}\left[\log \operatorname{tech}_{h t}-l_{h t}\right]+a
\end{gathered}
$$

où $l_{h t}=\log \left(\right.$ lit $\left._{h t}\right)$ et $q_{h t}=\log \left(Q_{h t}\right)$.

Le terme $a$ désigne une constante et $\mu$ est le paramètre des rendements d'échelle. Comme nous l'avons expliqué dans l'analyse descriptive, des problèmes d'observabilité font qu'il est préférable de traiter les médecins comme une variable omise. En formalisant l'hétérogénéité non observée propre à l'hôpital et son inefficacité productive, la spécification économétrique s'écrit alors :

$$
\begin{gathered}
q_{h t}-l_{h t}=(\mu-1) l_{h t}+\alpha_{2}\left[\log i n f_{h t}-l_{h t}\right]+\alpha_{3}\left[\log a s_{h t}-l_{h t}\right]+\alpha_{4}\left[\log a d m_{h t}-l_{h t}\right]+ \\
\alpha_{5}\left[\log t e c h_{h t}-l_{h t}\right]+c_{t}+\gamma+\delta c h u_{h}+v_{h}-u_{h}+\xi_{h t}
\end{gathered}
$$

$\gamma$ est une constante et $c_{t}$ un effet fixe spécifique à l'année. Une variable $c h u_{h}$ indique si l'hôpital est un centre hospitalo-universitaire. $v_{h}$ est une variable aléatoire mesurant l'hétérogénéité non observée au niveau de l'hôpital et $u_{h} \geq 0$ est une variable aléatoire positive ou nulle qui permet d'évaluer la distance à la frontière de production, c'est-à-dire l'inefficacité productive de l'hôpital. $\xi_{h t}$ est une perturbation supposée i.i.d. $\left(0, \sigma_{\xi}^{2}\right)$ et non corrélée avec les variables explicatives du modèle.

\section{Estimation}

On utilise une approche en deux étapes pour l'estimation. La première étape consiste à appliquer les moindres carrés ordinaires à la spécification :

$$
\begin{gathered}
q_{h t}-l_{h t}=(\mu-1) l_{h t}+\alpha_{2}\left[\log i n f_{h t}-l_{h t}\right]+\alpha_{3}\left[\log a s_{h t}-l_{h t}\right]+\alpha_{4}\left[\log a d m_{h t}-l_{h t}\right]+ \\
\alpha_{5}\left[\log t e c h_{h t}-l_{h t}\right]+c_{t}+\eta_{h}+\xi_{h t}
\end{gathered}
$$

où $\eta_{h}$ est un effet fixe propre à l'hôpital $h$. 
Dans la seconde étape, on utilise une approche en termes de frontière stochastique (Aigner et al, 1977, Jondrow et al., 1982) pour décomposer les effets fixes hôpitaux en estimations de I'hétérogénéité non observée $v_{h}$ d'une part, de l'inefficacité productive de l'hôpital $u_{h}$, d'autre part.

Plus exactement on a, d'après (3) :

$$
\hat{\eta}_{h}=\gamma+\delta \cdot \operatorname{ch} u_{h}+v_{h}-u_{h}
$$

Cette équation est estimée par le maximum de vraisemblance. Le modèle est dit normal-demi normal si on suppose que $v_{h} \sim N\left(0, \sigma_{v}^{2}\right)$ et $u_{h} \sim N^{+}\left(0, \sigma_{u}^{2}\right)$. Une autre possibilité consiste à supposer que $u_{h}$ suit une distribution exponentielle, auquel cas le modèle est normal-exponentiel. La distance entre la production effectivement réalisée $q_{h}$ et la production potentielle $q_{h}^{\max }$ donnée par la frontière est mesurée par $u_{h}$. L'estimation permet de calculer un paramètre d'asymétrie $\lambda=\frac{\sigma_{u}}{\sigma_{v}}$ qui évalue l'importance de l'inefficacité dans les composantes de l'inobservé. On peut en outre calculer, pour chaque hôpital, un taux d'efficacité productive effi $i_{h}$ défini par :

$$
\text { effi } i_{h}=\exp \left\{-u_{h}\right\}=\frac{Q_{h}}{Q_{h}^{\max }}
$$

Notre stratégie empirique consiste à appliquer cette estimation en deux étapes à trois modèles. Le premier est une fonction de production classique mettant en rapport inputs et output :

$$
q_{h t}-l_{h t}=x_{h t}^{\prime} \alpha+c_{t}+\eta_{h}+\xi_{h t}
$$

On considère ensuite des spécifications incorporant des variables explicatives additionnelles décrivant les caractéristiques des patients et de la production des hôpitaux :

$$
\begin{gathered}
q_{h t}-l_{h t}=x_{h t}^{\prime} \alpha+z_{h t}^{\prime} \beta+{c^{\prime}}_{t}+\eta_{h}^{\prime}+\xi_{h t}^{\prime} \\
q_{h t}-l_{h t}=x_{h t}^{\prime} \alpha+z_{h t}^{\prime} \beta+\pi_{h t}^{\prime} \theta+c^{\prime \prime}{ }_{t}+\eta^{\prime \prime}{ }_{h}+\xi^{\prime \prime}{ }_{h t}
\end{gathered}
$$

Le modèle (II) incorpore un vecteur $z_{h t}^{\prime}$ de 19 composantes décrivant les caractéristiques de la patientèle de l'hôpital : proportion de patients dans les différentes catégories d'âge, croisées avec le sexe, sévérité du séjour, mode d'entrée et de sortie de l'hôpital (par transfert ou non, retour ou non au domicile, occurrence d'un décès).

Le modèle (III) intègre un vecteur de 13 composantes $\pi_{h t}^{\prime}$ caractérisant l'activité de l'hôpital: proportion de séjours réalisés dans 9 importantes Catégories Majeures de Diagnostic, proportion de séjours en hospitalisation partielle (de moins de $24 \mathrm{H}$ ), degré de spécialisation et proportion de séjours chirurgicaux. Les CMD ont été sélectionnées sur la base d'une proportion de séjours supérieure à $\mathrm{x} \%$. II s'agit de la CMD 1, Affections du système nerveux, de la CMD 2, Affections de l'œil, de la CMD 3, Affections des oreilles, du nez, de la gorge, de la bouche et des dents, de la CMD 4, Affections de l'appareil respiratoire, de la CMD 5, Affections de l'appareil circulatoire, de la CMD 6, Affections du tube digestif, de la CMD 8, Affections et traumatismes de l'appareil musculosquelettique et du tissu conjonctif, de la CMD 14, Grossesses pathologiques, accouchements et 
affections du post-partum et de la CMD 23, Facteurs influant sur l'état de santé et autres motifs de recours aux services de santé.

L'approche que nous utilisons pour l'estimation est relativement spécifique. En effet, la plupart des travaux consacrés à des analyses de frontières stochastiques utilisent un estimateur du maximum de vraisemblance en une étape. Une telle approche n'est légitime que si l'on peut supposer que l'hétérogénéité non observée spécifique à l'hôpital $v_{h}$ et son inefficacité productive $u_{h}$ sont non corrélés aux variables explicatives du modèle. Cette hypothèse ne peut être retenue dans notre cas : $v_{h}$ est lié à différentes variables omises comme la qualité des soins ou le nombre de médecins, qui sont vraisemblablement corrélées au niveau des autres inputs. Notre stratégie repose sur l'hypothèse que les estimations de première étape des $\eta_{h}$ sont convergentes, ce qui est loin d'être assuré. Cela présente l'avantage toutefois d'éviter d'avoir à supposer que les variables aléatoires $v_{h}$ et $u_{h}$ sont non corrélées aux régresseurs.

A la suite de Battese et Coelli $(1992,1995)$, de nombreux travaux formalisent l'idée que le terme d'inefficacité $u_{h}$ est corrélé au temps et à certaines variables (Rosko, 2001 ; Herr et al., 2010). Mais il n'y a pas de discussion sur les raisons justifiant l'exclusion de ces variables de la liste des régresseurs de la fonction de production. Aucun critère n'est avancé pour expliquer pourquoi ces variables devraient expliquer l'efficacité plutôt que la production de l'hôpital.

Considérons par exemple les variables $z_{h t}^{\prime}$ : dans le modèle (II), elles sont ajoutées aux régresseurs $x_{h t}^{\prime}$ pour expliquer la productivité de l'hôpital. Une autre spécification pourrait être adoptée, qui consisterait à supposer que les variables $z_{h t}^{\prime}$ expliquent l'inefficacité (Battese et Coelli, 1995). Dans le premier cas, ces variables influencent la frontière, dans le second elles influencent la distance à la frontière. Notre but est d'intégrer des éléments liés au cahier des charges des hôpitaux publics dans la spécification de la fonction de production. Le fait qu'ils ne soient pas autorisés à sélectionner leurs patients, par exemple, doit conduire à des configurations spécifiques pour les valeurs prises par le vecteur $z_{h t}^{\prime}$, lequel reflète la composition de la patientèle en matière d'âge, de sexe et de sévérité des cas. Nous voulons examiner dans quelle mesure l'évaluation de l'efficacité de l'hôpital est influencée par la spécification de la frontière. Le modèle (I) est une fonction de production classique. Dans le modèle (II), on ajoute les caractéristiques des patients $z^{\prime}{ }_{h t}$ à la spécification de la frontière. Si le modèle (II) est la bonne spécification, estimer le modèle (I) par une méthode de maximum de vraisemblance en une étape en supposant que $v_{h}$ et $u_{h}$ sont aléatoires et non corrélés ne permet pas d'obtenir une estimation convergente si les $z_{h t}^{\prime}$, qui sont des variables omises dans (I), sont corrélés aux $x_{h t}^{\prime}$. Estimer un modèle à effets fixes permet d'éviter ces biais de variables omises.

Formellement, expliquer l'efficacité par des variables $z^{\prime}{ }_{h t}$, ou intégrer ces variables aux régresseurs de la fonction de production conduit à des spécifications très proches. Conceptuellement les deux approches ne sont pas très différentes non plus: dans un cas, on considère que la frontière des hôpitaux dépend des caractéristiques de leurs patients, dans l'autre on suppose qu'une partie de l'inefficacité peut être justifiée par ces mêmes caractéristiques. La question importante est celle de la corrélation ou non des termes $v_{h}$ et $u_{h}$ avec les variables explicatives du modèle. S'ils sont corrélés, il faut retenir une spécification avec des effets fixes et il est préférable de faire figurer les variables $z^{\prime}{ }_{h t}$ dans les régresseurs de la fonction de production. 


\section{Robustesse}

Les taux d'efficacité productive estimés à partir du modèle (I) font apparaître les hôpitaux publics comme moins efficaces que les hôpitaux privés, quelle que soit leur taille (tableau 4b), mais la situation relative des hôpitaux publics s'inverse lorsque l'on intègre les caractéristiques des patients (modèle (II)), et les caractéristiques de l'activité des hôpitaux. Avec le modèle (III), les hôpitaux publics apparaissent comme les plus efficaces, du moins en ce qui concerne les établissements de moyenne ou de grande taille.

Ce résultat concernant la hiérarchie des taux d'efficacité est obtenu de façon robuste pour différentes variantes: si l'on utilise une fonction de production Translog (plus flexible que la CobbDouglas), si l'on élimine les centres hospitalo-universitaires des données (tableaux 7 et 8 en annexe), si l'on estime les modèles sur l'échantillon restreint de 1169 hôpitaux (5 798 observations) pour lesquels on observe les médecins (dans ce cas en ne traitant pas les médecins comme une variable omise), si l'on élimine les hôpitaux locaux et les hôpitaux "hybrides" (nous qualifions ainsi des établissements qui ont beaucoup de séjours longs bien que figurant dans la base PMSI).

\section{Décompositions}

L'estimation de la fonction de production (tableau 3) permet de quantifier les composantes des différences de productivité entre les hôpitaux selon leur statut. Ces décompositions utilisent les coefficients estimés et les moyennes des variables explicatives, calculées pour chaque catégorie de statut et de taille d'hôpitaux. Trois statuts (publics, privés et PSPH) et trois tailles sont considérés, ce qui conduit à neuf combinaisons pour les contrastes (tableau 5). Comme la procédure en deux étapes est non linéaire, la décomposition fait apparaître un résidu, dont l'ampleur est négligeable, sauf pour les petits hôpitaux publics. 
Annexe :

Tableau 6 : Décomposition des différences de productivité (à partir du modèle I)

\begin{tabular}{|c|c|c|c|c|c|c|c|c|c|}
\hline & $\begin{array}{c}\text { Petit } \\
\text { Public - Privé } \\
\text { (a) }\end{array}$ & $\begin{array}{c}\text { Moyen } \\
\text { Public - Privé } \\
\text { (b) }\end{array}$ & $\begin{array}{c}\text { Grand } \\
\text { Public - Privé } \\
\text { (c) }\end{array}$ & $\begin{array}{c}\text { Petit } \\
\text { Public-PSPH } \\
\text { (d) }\end{array}$ & $\begin{array}{c}\text { Moyen } \\
\text { Public - PSPH } \\
\text { (e) }\end{array}$ & $\begin{array}{c}\text { Grand } \\
\text { Public - PSPH } \\
\text { (f) }\end{array}$ & $\begin{array}{c}\text { Petit } \\
\text { PSPH-Privé } \\
\text { (g) }\end{array}$ & $\begin{array}{c}\text { Moyen } \\
\text { PSPH-Privé } \\
\text { (h) }\end{array}$ & $\begin{array}{c}\text { Grand } \\
\text { PSPH-Privé } \\
\text { (i) }\end{array}$ \\
\hline $\begin{array}{c}\text { Différence } \\
\text { moyenne de } \\
\text { productivité } \\
\text { (à expliquer) (1) } \\
\end{array}$ & -54.5 & -33.6 & -33.7 & -37.2 & -26.2 & -12.7 & - 17.3 & - 7.4 & -21.0 \\
\hline $\begin{array}{l}\text { Due à : } \\
\text { Lits } \\
\text { Infirmières } \\
\text { Aide-soignantes } \\
\text { Pers. administratif } \\
\text { Pers. Medico-tech. } \\
\end{array}$ & $\begin{array}{l}+16.3 \\
+13.1 \\
+4.1 \\
+11.8 \\
-17.5 \\
\end{array}$ & $\begin{array}{l}-8.5 \\
+7.7 \\
+1.7 \\
+5.7 \\
-9.8 \\
\end{array}$ & $\begin{array}{l}-26.0 \\
+7.7 \\
+1.1 \\
+6.0 \\
-10.6 \\
\end{array}$ & $\begin{array}{l}+16.6 \\
+5.8 \\
+3.1 \\
+3.8 \\
-9.0 \\
\end{array}$ & $\begin{array}{l}+0.3 \\
+3.1 \\
+1.3 \\
-1.7 \\
-2.4 \\
\end{array}$ & $\begin{array}{l}-11.1 \\
+2.9 \\
+0.8 \\
-2.3 \\
-2.1 \\
\end{array}$ & $\begin{array}{l}-0.3 \\
+7.3 \\
+1.0 \\
+8.0 \\
-8.5 \\
\end{array}$ & $\begin{array}{l}-8.8 \\
+4.7 \\
+0.3 \\
+7.4 \\
-7.4 \\
\end{array}$ & $\begin{array}{l}-14.9 \\
+4.8 \\
+0.3 \\
+8.3 \\
-8.5 \\
\end{array}$ \\
\hline $\begin{array}{l}\text { Diff. totale due } \\
\text { aux facteurs de } \\
\text { production (2) } \\
\end{array}$ & +27.8 & -3.2 & -21.7 & +20.3 & +0.6 & -11.8 & +7.5 & -3.8 & -9.9 \\
\hline CHU (5) & +2.8 & +1.7 & +15.0 & +2.8 & +1.7 & +15.0 & 0 & 0 & 0 \\
\hline $\begin{array}{c}\text { Hétérogénéité } \\
\text { non observée }(6)\end{array}$ & -3.2 & -10.5 & -23.6 & -3.1 & -10.6 & -16.1 & -0.2 & +0.1 & - 7.5 \\
\hline Inefficacité (7) & -99.9 & -22.6 & -12.3 & -72.8 & -20.6 & -5.1 & -27.1 & -2.0 & -7.3 \\
\hline Résidu* (8) & +18.2 & +1.0 & +9.0 & +15.6 & +2.7 & +5.3 & +2.6 & -1.7 & +3.7 \\
\hline
\end{tabular}

1604 hôpitaux, 7731 observations dans la dimension hôpital-année ; période 1998-2003. On a : (g) = (a)-(d); (h) = (b)- (e); (i) = (c) - (f).

Un résidu apparaît car la procédure en deux étapes est non linéaire. 
Tableau 7. Robustesse des résultats : estimation de la première étape sans les CHU Variable dépendante: Log (production par lit)

\begin{tabular}{|c|c|c|}
\hline & \multicolumn{2}{|c|}{ Modèle I Modèle II } \\
\hline $\log ($ lit $)$ & $-0,3287^{* * *}$ & $-0,4852^{* * *}$ \\
\hline Log (infirmière/ lit) & $0,2937^{* * *}$ & $0,2108^{* * *}$ \\
\hline Log (aide soignante/lit) & 0,0328 & $0,0976^{*}$ \\
\hline Log (administratif/lit) & $0,4707^{\star * *}$ & $0,4187^{* * *}$ \\
\hline Log (médico-technique/lit) & $-0,3043^{* * *}$ & $-0,2604^{* * *}$ \\
\hline$\%$ femmes $19-40$ ans & & 0,2542 \\
\hline$\%$ hommes $19-40$ ans & & $0,9248^{* *}$ \\
\hline$\%$ femmes 41-50 ans & & 0,0309 \\
\hline$\%$ hommes $41-50$ ans $=$ ref. & & - \\
\hline$\%$ femmes 51-60 ans & & $-0,0615$ \\
\hline$\%$ hommes 51-60 ans & & 0,3755 \\
\hline$\%$ femmes $61-70$ ans & & $0,7794^{\star *}$ \\
\hline$\%$ hommes 61-70 ans & & 0,3745 \\
\hline$\%$ femmes $71-80$ ans & & 0,4453 \\
\hline$\%$ hommes 71-80 ans & & $-0,1212$ \\
\hline$\%$ femmes $81-90$ ans & & $-0,7391^{* * *}$ \\
\hline$\%$ hommes 81-90 ans & & $-0,0918$ \\
\hline$\%$ femmes 91 ans+ & & $-0,0136$ \\
\hline$\%$ hommes 91 ans+ & & $-0,8356$ \\
\hline \% séjours de sévérité $1=$ ref. & & - \\
\hline \% séjours de sévérité 2 & & $0,8225^{* \star *}$ \\
\hline \% séjours de sévérité 3 & & $1,6106^{* * *}$ \\
\hline Admission par transfert $=$ ref. & & - \\
\hline Admission en provenance du domicile & & $-0,1231^{* *}$ \\
\hline Sortie vers un autre service $=$ ref. & & - \\
\hline Sortie vers le domicile & & $-0,0460$ \\
\hline Sortie vers un autre hôpital & & $-0,0725$ \\
\hline Sortie par décès & & $-1,0490^{* * *}$ \\
\hline
\end{tabular}


Tableau 7 (suite)

\begin{tabular}{|c|c|}
\hline & Modèle I Modèle II \\
\hline \% séjours en CMD 1 & $-0,1739$ \\
\hline \% séjours en $C M D 2$ & $-0,1442$ \\
\hline \% séjours en $C M D 3$ & $-0,5621^{* *}$ \\
\hline \% séjours en CMD 4 & $0,7546^{* * *}$ \\
\hline \% séjours en CMD 5 & $0,8142^{\star \star *}$ \\
\hline \% séjours en CMD 6 & $1,6991^{* * *}$ \\
\hline \% séjours en CMD 8 & $0,4825^{\star \star}$ \\
\hline \% séjours en CMD 14 & $2,0743^{\star \star \star}$ \\
\hline \% séjours en CMD 23 & $0,3651^{* *}$ \\
\hline \% séjours en hosp. partielle (moins de 24h) & $0,7983^{* * *}$ \\
\hline \% séjours chirurgicaux & $0,9628^{* * *}$ \\
\hline Indice de spécialisation & $0,1887^{* *}$ \\
\hline Intensité de spécialisation & $-0,6530^{* * *}$ \\
\hline $\mathrm{R}^{2}$ & 0,99 \\
\hline
\end{tabular}

Echantillon sans les CHU: 1533 hôpitaux 7479 observations dans la dimension année-hôpital; période 1998-2003. MCO appliqués à des spécifications comprenant des effets fixes hôpitaux et des effets fixes temporels (années).

*: significatif (10\%), **: significatif $(5 \%)$, ***: significatif $(1 \%)$.

CMD 1: Affections du système nerveux CMD 2: Affections de l'œil CMD 3: Affections des oreilles, du nez, de la gorge, de la cavité buccale et des dents, CMD 4: affections de l'appareil respiratoire, CMD 5: Affections de l'appareil circulatoire CMD 6: affections du système digestif, CMD 8: Affections de l'appareil musculosquelettique et du tissu conjonctif CMD 14: Grossesse, accouchement et post partum CMD 23: Facteurs influant l'état de santé et autres motifs de recours aux services de santé. Les séjours en hospitalisation partielle (moins de 24H) correspondent souvent à de la chirurgie réalisée en ambulatoire.

Indice de spécialisation $=1 \mathrm{~s}$ 'il existe plus de $33 \%$ des séjours au sein du même hôpital dans une CMD donnée, l'intensité est égale à la valeur de cette proportion, si l'indice de spécialisation $=1$, à 0 sinon. 


\section{Tableau 9a}

Robustesse des résultats : estimation sans les CHU

Estimation de l'équation $\hat{\eta}_{h}=\gamma+v_{h}-u_{h}$

\begin{tabular}{|c|c|c|c|}
\hline & Modèle I & Modèle II & Modèle III \\
\hline \hline $\begin{array}{c}\text { Paramètre d'asymétrie } \\
\lambda=\frac{\sigma_{u}}{\sigma_{v}}\end{array}$ & 3.434 & 2.725 & 1.127 \\
\hline \hline $\begin{array}{c}\text { Risque de première espèce } \\
\text { pour le test de rapport de } \\
\text { vraisemblance de } \mathrm{H}_{0}: \sigma_{u}=0\end{array}$ & 0.000 & 0.000 & 0.000 \\
\hline \hline
\end{tabular}

\section{Tableau 9b}

Médianes des taux d'efficacité productive estimés eff $_{\boldsymbol{h}}$

\begin{tabular}{|c|c|c|c|c|}
\hline Taille & Statut & Modèle I & Modèle II & Modèle III \\
\hline \hline \multirow{3}{*}{ Petit } & Public & 18.1 & 35.5 & 54.2 \\
& PSPH & 43.9 & 50.7 & 66.0 \\
& Privé & 58.3 & 57.2 & 64.5 \\
\hline \hline \multirow{3}{*}{ Moyen } & Public & 64.9 & 75.4 & 79.2 \\
& PSPH & 79.5 & 75.8 & 79.0 \\
& Privé & 81.0 & 80.5 & 77.0 \\
\hline \hline \multirow{3}{*}{ Grand } & Public & 83.7 & 87.6 & 85.5 \\
& PSPH & 87.6 & 85.6 & 84.1 \\
& Privé & 88.8 & 87.6 & 82.0 \\
\hline
\end{tabular}

Echantillon sans les CHU: 1533 hôpitaux 7479 observations dans la dimension année-hôpital;

période 1998-2003. Le taux d'efficacité est défini pour chaque hôpital $h$ par $\operatorname{effi} i_{h}=\exp \left(-u_{h}\right)=Q_{h} / Q^{\max }{ }_{h}$

Lecture : effi $=83.7$ est la médiane des effi $i_{h}$ estimés pour les grands hôpitaux publics : en valeur médiane, les grands hôpitaux publics produisent seulement $83.7 \%$ de ce qu'ils pourraient produire, compte tenu des facteurs utilisés. 